\title{
Design, Synthesis and Comparative Study of Anti-Microbial Activities on Barbituric Acid and Thiobarbituric Acid based Chalcone Derivatives Bearing the Pyrimidine Nucleus
}

Dhorajiya BD, Bhuva RG and Dholakiya BZ*

Department of Applied Chemistry, SV National Institute of Technology, Ichchhanath, Surat-395 007, Gujarat, India

\begin{abstract}
A new comparative series of barbituric acid and thiobarbituric acid based chalcone derivatives bearing the pyrimidine nucleus were synthesized. The chemical structures of the resulting molecules were characterised by means of FT-IR (Fourier Transform Infrared) ${ }^{1} \mathrm{H}$ NMR, ${ }^{13} \mathrm{C}-\mathrm{NMR}$ (Nuclear Magnetic Resonance) and HMBC (Heteronuclear Multiple-Bond Correlation) and Elemental Analysis. All synthesized compounds were subjected to in vitro antimicrobial screening against four bacterial strains i.e., one Gram Positive (Bacillus subtilis MTCC 441), two Gram Negative (E. coli MTCC 443, P. aeruginosa MTCC 1688), and one Fungal (C. albicans MTCC 227) Strains. The structure activity relationship is discussed on the basis of bioactivity results, various functional groups present and position of the functional group at various positions of the synthesized compounds. The comparative antimicrobial activity study of both of the series elucidated that shows the chalcone compounds containing -thioketo group are more potent than the -keto group.
\end{abstract}

Keywords: Antibacterial activity; Antifungal activity; Babiturates; Chalcones; Structure activity relationship

\section{Introduction}

Development of new antimicrobial agents with novel structure and mode of action remains the primary goal of scientists for the solution of increasing bacterial resistance gained by microorganism to classical antimicrobial agents [1]. As resistance to antimicrobial drugs is widespread, there is an increasing need for identification of novel structure leads that may be of use in designing new, potent and less toxic antimicrobial agents [2]. The multiple pharmacological actions of unique synthetic compounds are a prerequisite for classifying a drug as highly efficacious, because these actions offer possibility of treating various diseases. Pyrimidine derivatives are synthesized by the reaction of 5-acetyl barbituric acid or 5-acetyl thiobarbituric acid and various aldehydes in alkaline condition at room temperature. These derivatives are considered to be important for drugs [3]. The presence of a pyrimidine base in thymine, cytosine and uracil, which are the essential binding blocks of nucleic acids, DNA and RNA is one possible reason for their activity. The literature survey indicated that compounds having pyrimidine nucleus possess broad range of biological activities, like 5-fluorouracil as anticancer, idoxuridine and trifluridine as antiviral [4-6] zidovudine and stavudine as anti- HIV [7] trimethoprim, sulphamethiazine and sulphadiazine as antibacterial [8] sulphadoxin as antimalarial and antibacterial [9]. Fungi are widely distributed in nature and frequently appear as pathogens in the animal and plant kingdoms. The pyrimidine ring system being present in various natural compounds such as nucleic acids, vitamins, coenzymes, uric acid, purines, some marine microorganisms [10]. The therapeutic importance of pyrimidine motif- derivatives such as barbituric acid and thiobarbituric acid play vital role among various heterocyclic compounds due to their anti-neoplastic [11,12], antiviral [13], antibiotic [14], and anti-inflammatory [11] activity. Many synthetic drugs of barbituric and thiobarbituric acid motif based derivatives and chemotherapeutic agents are well known [15]. Chalcones are the products of the condensation of a simple or substituted aromatic moiety with a simple or substituted acetophenone in presence of base. This group of compounds is widely used in anticancer research, as an antimicrobial or an antitubercular [16,17]. Chalcone analogues are very versatile as physiologically active compounds and substrates for the evaluation of various organic syntheses. So, in light of above facts of pyrimidine, barbituric acid, thiobarbituric acid and chalcones, we continue our earlier work on synthesis of 5-acetyl barbituric acid 4 and 5-acetyl thiobarbituric acid 4' based chalcones 5 (a-k) and $5\left(\mathbf{a}^{\prime}-\mathbf{k}^{\prime}\right)$. All the analogs were screened for their antimicrobial activity and their comparative results are discussed with respect to one Gram Positive (Bacillus subtilis MTCC 441), two Gram Negative (E. coli MTCC 443, $P$. aeruginosa MTCC 1688), and one Fungal (C. albicans MTCC 227) Species and effects of functional groups and position of functional groups on various microbial strains.

\section{Experimental Methods}

Chemicals and solvents were obtained from commercial sources and used as received throughout the investigation. Melting points were determined in open capillaries on a Veego electronic apparatus VMP-D (Veego Instrument Corporation, Mumbai, India) and are uncorrected. IR spectra (4000-400 $\mathrm{cm}^{-1}$ ) of synthesized compounds were recorded on a Perkin Elmer-Spectrum RX-IFTIR spectrophotometer using $\mathrm{KBr}$ pellets. Thin layer chromatography was performed on object glass slides $(2 \times 7.5 \mathrm{~cm})$ coated with silica gel-G and spots were visualized under UV irradiation. ${ }^{1} \mathrm{H}$ NMR and ${ }^{13} \mathrm{C}$ NMR spectra were recorded on an Advance-II (Bruker) model using DMSO as a solvent and TMS as internal standard with ${ }^{1} \mathrm{H}$ resonant frequency of $400 \mathrm{MHz}$ and ${ }^{13} \mathrm{C}$ resonant frequency of $100 \mathrm{MHz}$. The ${ }^{1} \mathrm{H}$ NMR and ${ }^{13} \mathrm{C}$ NMR chemical shifts were reported as parts per million (ppm) downfield from TMS

*Corresponding author: Bharathkumar Z Dholakiya, Department of Applied Chemistry, SV National Institute of Technology, Ichchhanath, Surat-395 007, Gujarat India, Tel: +919428949595; Fax: +910261227334; E-mail: bharat281173@gmail.com

Received April 26, 2016; Accepted May 06, 2016; Published May 12, 2016

Citation: Dhorajiya BD, Bhuva RG, Dholakiya BZ (2016) Design, Synthesis and Comparative Study of Anti-Microbial Activities on Barbituric Acid and Thiobarbituric Acid based Chalcone Derivatives Bearing the Pyrimidine Nucleus. Chem Sci J 7: 126 doi:10.4172/2150-3494.1000126

Copyright: (c) 2016 Dhorajiya BD, et al. This is an open-access article distributed under the terms of the Creative Commons Attribution License, which permits unrestricted use, distribution, and reproduction in any medium, provided the original author and source are credited. 
$\left(\mathrm{Me}_{4} \mathrm{Si}\right)$. The splitting patterns are designated as follows; s, singlet; br s, broad singlet; d, doublet; $\mathrm{t}$, triplet; q, quartet; $\mathrm{m}$, multiplet. All standard strains for screening of antibacterial and antifungal activities were procured from Institute of Microbial Technology, Chandigarh. DMSO was used as diluents/vehicle to get desired concentration of drugs to test upon Standard bacterial strains.

\section{Synthesis of barbituric acid (3)}

To a solution of diethylmalonate $\mathbf{1}(20 \mathrm{~g}, 118.9 \mathrm{mmol})$, urea 2 in methanol, anhydrous sodium methoxide was added and refluxed at $65^{\circ} \mathrm{C}$ for $8 \mathrm{~h}$. A white solid separates. Then in above reaction mixture $125 \mathrm{ml}$. of hot $\left(50^{\circ} \mathrm{C}\right)$ water was added and hydrochloric acid was used to make the solution acidic. After completion of the reaction, the resulting clear solution was filtered and cooled in an ice bath overnight. The white product formed and it was filtered, washed with $50 \mathrm{ml}$ of cold water, dried and recrystallized from acetone to afford compound $\mathbf{3}$ as a white powder [18-20].

\section{Synthesis of thiobarbituric acid (3')}

To a solution of diethylmalonate $\mathbf{1}(20 \mathrm{~g}, 118.9 \mathrm{mmol})$, thiourea 2' (7.5 g, $125 \mathrm{mmol}$ ) in methanol, anhydrous sodium methoxide was added and refluxed at $65^{\circ} \mathrm{C}$ for $8 \mathrm{~h}$. A white solid separates. Then in above reaction mixture $125 \mathrm{ml}$ of hot $\left(50^{\circ} \mathrm{C}\right)$ water was added and hydrochloric acid was used to make the solution acidic. After completion of the reaction, the resulting clear solution was filtered and cooled in an ice bath overnight. The white product formed and it was filtered, washed with $50 \mathrm{ml}$ of cold water, dried and recrystallized from acetone to afford compound 3' as a Light yellowish white powder [18-20].

\section{Synthesis of 5-acetyl barbituric acid (4)}

To a solution of barbituric acid (3) $(6.4 \mathrm{~g}, 44.39 \mathrm{mmol})$ in acetic anhydride $(150 \mathrm{ml})$, few drops of $\mathrm{H}_{2} \mathrm{SO}_{4}$ was added and refluxed for 1 $\mathrm{h}$. The reaction in the beginning was a suspension but after about 10 min of refluxed, it changes to orange /brown color clear solution. The reaction mixture was concentrated into $1 / 2$ of its original volume and cooled at about $10^{\circ} \mathrm{C}$. The solid product was formed, filtered, washed with hot water then acetone, and dried to give compound $\mathbf{4}$ as a yellow powder [21].

\section{Synthesis of 5-acetyl thiobarbituric acid (4')}

To a solution of barbituric acid (3) and thiobarbituric acid (3') $(6.4 \mathrm{~g}, 44.39 \mathrm{mmol})$ in acetic anhydride $(150 \mathrm{ml})$, few drops of $\mathrm{H}_{2} \mathrm{SO}_{4}$ was added and refluxed for $1 \mathrm{~h}$. The reaction in the beginning was a suspension but after about $10 \mathrm{~min}$ of refluxed, it changes to orange/ brown color clear solution. The reaction mixture was concentrated into $1 / 2$ of its original volume and cooled at about $10^{\circ} \mathrm{C}$. The solid product was formed, filtered, washed with hot water then acetone, dried to give compound $\mathbf{4}$ and $\mathbf{4}$ ' as a brown powder [21].

\section{General synthetic procedure for the barbituric acid based} chalcone compounds 5 (a-k)

To a well stirred solution of compound 5-acetyl barbituric acid (4) in $40 \%$ aqueous sodium hydroxide solution, equimolecular amount of the appropriate aldehydes were was added. The reaction mixture was stirred at room temperature for about $12 \mathrm{~h}$. The confirmation of the reaction was carried out by TLC using chloroform-methanol and Hexane-Ethyl acetate (4:1 v/v) mixture. After completion of the reaction, final compound was isolated from water at 6-7 $\mathrm{pH}$. Further purification of isolated compound was done by recrystallization in methanol. Similarly other compounds $\mathbf{5}(\mathbf{a}-\mathbf{k})$ were synthesized $[22,23]$.

\section{General synthetic procedure for the thiobarbituric acid based compounds 5 (a'-k')}

To a well stirred solution of compound 5-acetyl thiobarbituric acid (4') in $40 \%$ aqueous sodium hydroxide solution, equimolecular amount of the appropriate aldehydes were was added. The reaction mixture was stirred at room temperature for about $12 \mathrm{~h}$. The confirmation of the reaction was carried out by TLC using chloroform-methanol and Hexane-Ethyl acetate $(4: 1 \mathrm{v} / \mathrm{v})$ mixture. After completion of the reaction, final compound was isolated from water at 6-7 $\mathrm{pH}$. Further purification of isolated compound was done by recrystallization in methanol. Similarly other compounds 5 ( $\left.\mathbf{a}^{\prime}-\mathbf{k}^{\prime}\right)$ were synthesized [22,23].

\section{Chemistry}

The synthetic strategy adopted to obtain the target compounds were depicted in Scheme 1. Diethylmalonate on reaction with urea and thiourea at $65^{\circ} \mathrm{C}$ yielded barbituric acid and thiobarbituric acid. The key intermediate 5-acetyl barbituric acid and 5-acetyl thiobarbituric acid were prepared in an excellent yield by refluxing compound barbituric acid and thiobarbioturic acid with acetic anhydride in the presence of $\mathrm{H}_{2} \mathrm{SO}_{4}$ respectively. Compounds such as 5-acetyl barbituric acid and 5 -acetyl thiobarbituric acid were treated with aromatic aldehydes $(\mathrm{a}-\mathrm{k})$ gave the corresponding chalcone derivatives $\mathbf{5}(\mathbf{a}-\mathbf{k})$ and $\mathbf{5}\left(\mathbf{a}^{\prime}-\mathbf{k}\right.$ ').

\section{Results and Discussion}

\section{Spectral characteristics and tautomerism}

The structures of the synthesized compounds were confirmed by spectral data and elemental analysis and they were in full agreement with the proposed structures. The FT-IR spectra of compounds 5 (ak) and $\mathbf{5}\left(\mathbf{a}^{\prime}-\mathbf{k}^{\prime}\right)$ revealed a characteristic bands between $3010 \mathrm{~cm}^{-1}$ and $3085 \mathrm{~cm}^{-1}$ confirms the presence of $(C=C)$ groups. Furthermore, in the FT-IR spectra the bands between $1682-1697 \mathrm{~cm}^{-1}$ confirms the presence of $(C=O)$ group. Moreover, a characteristic band appeared at $2526-2544 \mathrm{~cm}^{-1}$ corresponded to the presence of $(C=S)$ groups. The FT-IR spectra of compounds $\mathbf{5 a}$ and $\mathbf{5 d}$ 'reveled peaks at $1512 \mathrm{~cm}^{-1}$

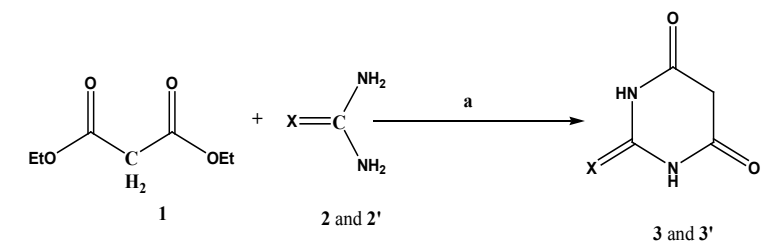

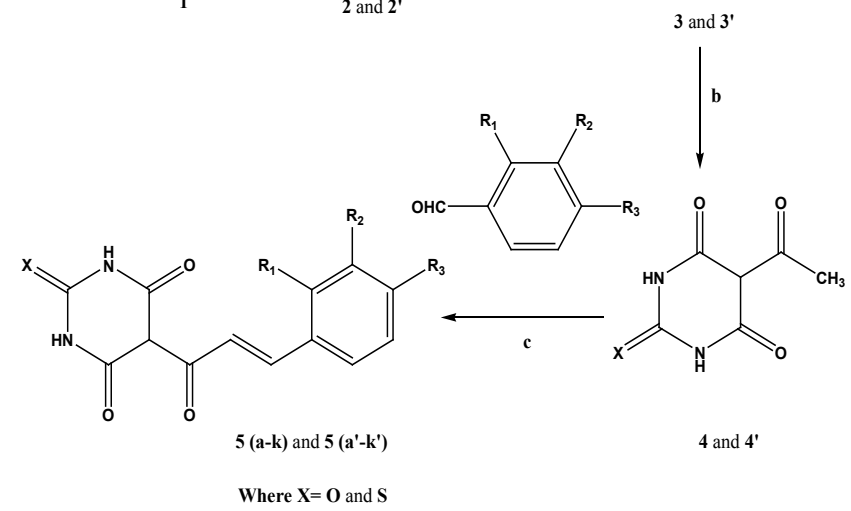

Reagents and Conditions: (a) NaOMe / MeOH-Refluxed (b) $\mathrm{AC}_{2} \mathrm{O}, \mathrm{H}_{2} \mathrm{SO}_{4}$ (c) Aqueous NaOH-Room Temperature

Scheme 1: Synthesis of compounds 5(a-k) and 5(a'-k'). 
Citation: Dhorajiya BD, Bhuva RG, Dholakiya BZ (2016) Design, Synthesis and Comparative Study of Anti-Microbial Activities on Barbituric Acid and Thiobarbituric Acid based Chalcone Derivatives Bearing the Pyrimidine Nucleus. Chem Sci J 7: 126. doi:10.4172/2150-3494.1000126

and $1522 \mathrm{~cm}^{-1}$, which shows partial $\boldsymbol{H}$-Bonding between $\mathrm{H}$-atom of olefinic carbon atom and $\mathrm{O}$-atom of pyrimidine ring. The ${ }^{1} \mathrm{H}$ NMR data of chalcone derivatives showed signals between $6.48-7.67 \delta \mathrm{ppm}$ for aromatic protons corresponding to the phenyl ring. The ${ }^{1} \mathrm{H}$ NMR data of compounds revealed signals between 7.08-11.73 $\delta \mathrm{ppm}-\mathrm{NH}$ of pyrimidine ring. From the ${ }^{1} \mathrm{H}$ NMR of compounds 5-acetyl barbituric acid and 5-acetyl thiobarbituric acid and its chalcones proves that when chalcones forms $-\mathrm{NH}$ protons of thee pyrimidine ring shifts towards the up field. Tautomeric study is important for other areas of chemistry. From ${ }^{1} \mathrm{H}$ NMR signal at $12.34-17.36 \delta$ ppm indicates the presence of $-\mathrm{OH}$ group proton which confirms the formation of tautomeric mixture but it is in the minor amount [24].

Tautomers not only have different colors, but also have different tinctorial strengths and different properties. Chalcones $\mathbf{5}(\mathbf{a}-\mathbf{k})$ and $\mathbf{5}$ (a'-k') can exist in different ten possible tautomeric forms, namely the T1, T2, T3, T4, T5 and T6 as shown in Figure 1.

Spectral Characterization data of synthesized compounds $5(a-k)$ and $5\left(a^{\prime}-k^{\prime}\right)$

5-(3-Phenyl-acryloyl) pyrimidine-2,4,6-trione (5a): Yellow Solid, M.W.258.23, Yield $89 \%$; m.p. $184-188^{\circ} \mathrm{C} ;{ }^{1} \mathrm{H}$ NMR (DMSO- $d_{6}$ ): $\delta 2.51$ $(2 \mathrm{H}, \mathrm{s}, J=23,8$ trans- $\mathrm{CH}=\mathrm{CH}), 4.16(1 \mathrm{H}, \mathrm{s},-\mathrm{CH}$ of pyrimidine ring at C-5), 7.40-7.93 (5H, m, Ar-H), $10.98(1 \mathrm{H}, \mathrm{s}$, barbituric acid $\mathrm{NH})$, $11.73\left(1 \mathrm{H}, \mathrm{s}\right.$, barbituric acid NH), ${ }^{13} \mathrm{C}$ NMR (DMSO- $\left.d_{6}\right): \delta 78.55(\mathrm{C}-$ 5), 116.12 (C-9), 116.73 (C-8), 129.13 (C-13), 130.70 (C-12, C-14), 129.13 (C-11, C-15), 132.38 (C-10), 167.29 (C-2, C-4, C-6, C-7); FT-IR $\left(\mathrm{KBr}, \mathrm{cm}^{-1}\right): 1136.11(\mathrm{C}-\mathrm{O}), 1612.21(\mathrm{C}=\mathrm{C}$ aromatic $), 1696.32(\mathrm{C}=\mathrm{O})$, $3438.52(\mathrm{~N}-\mathrm{H})$; Anal. Calcd. For $\mathrm{C}_{13} \mathrm{H}_{10} \mathrm{~N}_{2} \mathrm{O}_{4}$ : C 60.47, H 3.09, N 10.84 (\%). Found: C 60.45, H 3.05, N 10.87 (\%).

5-(3-Phenyl-acryloyl)-2-thioxo-dihydro-pyrimidine-4,6-dione (5a'): Yellow Solid, M.W.274.30, Yield 77\%; m.p. $>250^{\circ} \mathrm{C}$; ${ }^{1} \mathrm{H}$ NMR $\left(\mathrm{DMSO}-d_{6}\right): \delta 2.53(2 \mathrm{H}, \mathrm{s}, J=16.2$, trans-CH=CH), $3.82(1 \mathrm{H}, \mathrm{s},-\mathrm{CH}$ of pyrimidine ring at $\mathrm{C}-5), 6.49-7.39(5 \mathrm{H}, \mathrm{m}, \mathrm{Ar}-\mathrm{H}), 7.91(1 \mathrm{H}, \mathrm{s}$, barbituric acid $\mathrm{NH}), 8.14(1 \mathrm{H}, \mathrm{s}$, barbituric acid $\mathrm{NH}),{ }^{13} \mathrm{C}$ NMR (DMSO- $\left.d_{6}\right): \delta$ 77(C-5), 114.32 (C-9), 114.67 (C-8), 122.95 (C-13), 127.12(C-12, C-14),<smiles>[R]c1ccc(C(=O)N=c2[nH]c(O)c(C(=O)/C=C/c3ccccc3)c(=O)[nH]2)cc1</smiles><smiles>[R]c1ccc(/C=C/C(=O)C2C(=O)NC(=[X])NC2=O)cc1</smiles><smiles>[R]c1ccc(/C=C/C(=O)c2c(O)nc([Y])[nH]c2=O)cc1</smiles>

$\mathrm{T} 4$

$\mathrm{T} 5$

Figure 1: Possible tautomers of compounds 5(a-a) and 5(a'-k').
129.27 (C-11, C-15), 132 (C-10), 155(C-7), 163.15(C-4, C-6), 168.05 (C-2); FT-IR ( $\left.\mathrm{KBr}, \mathrm{cm}^{-1}\right): 1251.15(\mathrm{C}-\mathrm{SH}), 1612.12$ (C=C aromatic), 1696.23 (C=O), $3335.52(\mathrm{~N}-\mathrm{H})$; Anal. Calcd. For $\mathrm{C}_{13} \mathrm{H}_{10} \mathrm{~N}_{2} \mathrm{O}_{3} \mathrm{~S}: \mathrm{C} 56.92$, H 3.67, N 10.21 (\%). Found: C 56.89, H 3.71, N 10.19(\%).

5-[3-(2-Hydroxy-phenyl)-acryloyl]pyrimidine-2,4,6-trione (5b): Brown Solid, M.W.274.22, Yield 81\%; m.p. 293-296 ${ }^{\circ}$ C; ${ }^{1} \mathrm{H}$ NMR $\left(\mathrm{DMSO}-d_{6}\right): \delta 2.58(2 \mathrm{H}, \mathrm{s}, J=28$, trans- $\mathrm{CH}=\mathrm{CH}), 4.22(1 \mathrm{H}, \mathrm{s},-\mathrm{CH}$ of pyrimidine ring at C-5), 6.52-7.97 $(4 \mathrm{H}, \mathrm{m}, \mathrm{Ar}-\mathrm{H}), 10.61(1 \mathrm{H}, \mathrm{s}$, barbituric acid $\mathrm{NH}), 10.85(1 \mathrm{H}, \mathrm{s}$, barbituric acid $\mathrm{NH}) 6.52(1 \mathrm{H}, \mathrm{s}, \mathrm{o}-$ Ar- OH); ${ }^{13} \mathrm{C}$ NMR (DMSO- $d_{6}$ ): $\delta 77$ (C-5), 116.56 (C-9), $117.16(\mathrm{C}-8)$, 123.28 (C-13, C-14), 127.29 (C-12, C-15), 130.71 (C-11), 132.91 (C10) 156.23 (C-7), 167.08 (C-4, C-6), $169.52(\mathrm{C}-2)$; FT-IR $\left(\mathrm{KBr}, \mathrm{cm}^{-1}\right)$ : 1138.21 (C-O), $1614.24(\mathrm{C}=\mathrm{C}$ aromatic), $1698.32(\mathrm{C}=\mathrm{O}), 3437.23(\mathrm{~N}-$ $\mathrm{H}$ ); Anal. Calcd. For $\mathrm{C}_{13} \mathrm{H}_{10} \mathrm{~N}_{2} \mathrm{O}_{5}$ : C 56.94, H 3.68, N 10.22 (\%). Found (\%): 56.91, H 3.65, N 10.25 (\%).

5-[3-(2-Hydroxy-phenyl)-acryloyl]-2-thioxo-dihydropyrimidine-4,6-dione(5b'): Light Brown Solid, M.W. 290.29, Yield $58 \%$; m.p. $>250^{\circ} \mathrm{C}$; ${ }^{1} \mathrm{H}$ NMR (DMSO- $\left.d_{6}\right): \delta 2.51(2 \mathrm{H}, \mathrm{s}, J=16.1$, trans$\mathrm{CH}=\mathrm{CH}), 3.72(1 \mathrm{H}, \mathrm{s},-\mathrm{CH}$ of pyrimidine ring at $\mathrm{C}-5), 6.59-7.49(4 \mathrm{H}$, $\mathrm{m}, \mathrm{Ar}-\mathrm{H}), 7.89(1 \mathrm{H}, \mathrm{s}$, barbituric acid NH), $8.19(1 \mathrm{H}, \mathrm{s}$, barbituric acid NH) $9.29(1 \mathrm{H}, \mathrm{s}, \mathrm{o}-\mathrm{Ar}-\mathrm{OH}) ;{ }^{13} \mathrm{C}$ NMR (DMSO- $\left.d_{6}\right): \delta 76(\mathrm{C}-5), 115.10$ (C-9), 115.40 (C-8), 122.82 (C-13, C-14), 126.72(C-12, C-15), 129.67 (C-11), 131.89 (C-10) 155.15 (C-7), 165.15(C-4, C-6), 168.25 (C-2); FT-IR (KBr, $\left.\mathrm{cm}^{-1}\right): 1253.21$ (C-SH), $1608.14(\mathrm{C}=\mathrm{C}$ aromatic), 1693.36 $(\mathrm{C}=\mathrm{O}), 3339.27(\mathrm{~N}-\mathrm{H})$; Anal. Calcd. For $\mathrm{C}_{13} \mathrm{H}_{10} \mathrm{~N}_{2} \mathrm{O}_{4} \mathrm{~S}: \mathrm{C} 53.79, \mathrm{H} 3.47$, $\mathrm{N} 9.65$ (\%). Found (\%): 53.76, H 3.51, N 9.62(\%).

5-[3-(4-Hydroxy-phenyl)-acryloyl]pyrimidine-2,4,6-trione (5c): Yellow Solid, M.W. 274.22, Yield 79\%; m.p. 206-210 ${ }^{\circ} \mathrm{C} ;{ }^{1} \mathrm{H}$ NMR $\left(\mathrm{DMSO}-d_{6}\right): \delta 2.61(2 \mathrm{H}, \mathrm{s}, J=20$, trans- $\mathrm{CH}=\mathrm{CH}), 4.19(1 \mathrm{H}, \mathrm{s},-\mathrm{CH}$ of pyrimidine ring at C-5), 6.67-7.68 $(2 \mathrm{H}, \mathrm{dd}, \mathrm{Ar}-\mathrm{H}), 10.55(1 \mathrm{H}, \mathrm{s}$, barbituric acid $\mathrm{NH}), 10.69(1 \mathrm{H}, \mathrm{s}$, barbituric acid $\mathrm{NH}) 6.67(1 \mathrm{H}, \mathrm{s}, \mathrm{p}-$ Ar- OH); ${ }^{13} \mathrm{C}$ NMR (DMSO- $\left.d_{6}\right): \delta 79$ (C-5), 114.08 (C-9), 115.81 (C-8), 120.92 (C-13), 125.61 (C-12, C-14), 128.66 (C-11, C-15), 132.17 (C10), 158.52 (C-7), 164.67 (C-4, C-6), 167.35 (C-2); FT-IR $\left(\mathrm{KBr}, \mathrm{cm}^{-1}\right)$ : $1140.24(\mathrm{C}-\mathrm{O}), 1616.07$ (C=C aromatic), $1697.19(\mathrm{C}=\mathrm{O}), 3437.45(\mathrm{~N}-$ $\mathrm{H}$ ); Anal. Calcd. For $\mathrm{C}_{13} \mathrm{H}_{10} \mathrm{~N}_{2} \mathrm{O}_{5}: \mathrm{C} 56.94, \mathrm{H} 3.68, \mathrm{~N} 10.22$ (\%). Found: 56.97, H 3.72, N 10.24 (\%).

5-[3-(4-Hydroxy-phenyl)-acryloyl]-2-thioxo-dihydropyrimidine-4,6-dione (5c'): Dark Brown Solid, M.W. 290.29, Yield $82 \%$; m.p. $>250{ }^{\circ} \mathrm{C} ;{ }^{1} \mathrm{H}$ NMR (DMSO- $\left.d_{6}\right): \delta 2.54(2 \mathrm{H}, \mathrm{s}, J=16.4$, trans$\mathrm{CH}=\mathrm{CH}), 3.74(1 \mathrm{H}, \mathrm{s},-\mathrm{CH}$ of pyrimidine ring at $\mathrm{C}-5), 6.49-7.67(2 \mathrm{H}$, dd, Ar-H), $7.88(1 \mathrm{H}, \mathrm{s}$, barbituric acid NH), $8.15(1 \mathrm{H}, \mathrm{s}$, barbituric acid NH) $9.37(1 \mathrm{H}, \mathrm{s}, \mathrm{p}-\mathrm{Ar}-\mathrm{OH}) ;{ }^{13} \mathrm{C}$ NMR (DMSO- $\left.d_{6}\right): \delta 78(\mathrm{C}-5), 113.10$ (C-9), 113.38 (C-8), 121.39 (C-13), 126.56(C-12, C-14), 129.60(C-11, C-15), 133.29 (C-10), 157.55 (C-7), 163.15(C-4, C-6), 168.05 (C-2); FT-IR (KBr, $\left.\mathrm{cm}^{-1}\right): 1257.33(\mathrm{C}-\mathrm{SH}), 1614.17(\mathrm{C}=\mathrm{C}$ aromatic), 1689.15 $(\mathrm{C}=\mathrm{O})$, 3328.54(N-H); Anal. Calcd. For $\mathrm{C}_{13} \mathrm{H}_{10} \mathrm{~N}_{2} \mathrm{O}_{4} \mathrm{~S}$ : C 53.79, H 3.47, N 9.65 (\%). Found: 53.74, H 3.50, N 9.61(\%).

5-[3-(3-Methoxy-phenyl)-acryloyl]pyrimidine-2,4,6-trione (5d): Yellow Solid, M.W.288.66, Yield 83\%; m.p. $228-233^{\circ} \mathrm{C} ;{ }^{1} \mathrm{H}$ NMR $\left(\mathrm{DMSO}-d_{6}\right): \delta 2.62(2 \mathrm{H}, \mathrm{s}, J=12$, trans- $\mathrm{CH}=\mathrm{CH}), 4.20(1 \mathrm{H}, \mathrm{s},-\mathrm{CH}$ of pyrimidine ring at C-5), $3.09\left(3 \mathrm{H}, \mathrm{s}, \mathrm{m}-\mathrm{OCH}_{3}\right), 6.67-7.77(2 \mathrm{H}, \mathrm{dd}, \mathrm{Ar}-$ $\mathrm{H}), 10.62(1 \mathrm{H}, \mathrm{s}$, barbituric acid $\mathrm{NH}), 10.79(1 \mathrm{H}, \mathrm{s}$, barbituric acid $\mathrm{NH})$; ${ }^{13} \mathrm{C}$ NMR (DMSO- $d_{6}$ ): $\delta 57.87$ (C-18), 77.54 (C-5), 114.63 (C-9), 115.78 (C-8), 123.58 (C-11, C-15), 128.12 (C-12, C-14), 130.13 (C-10, C-13), 156.84 (C-7), 163.43 (C-4, C-6), 168.32 (C-2); FT-IR $\left(\mathrm{KBr}, \mathrm{cm}^{-1}\right)$ : $1136.78(\mathrm{C}-\mathrm{O}), 1613.24(\mathrm{C}=\mathrm{C}$ aromatic), $1698.87(\mathrm{C}=\mathrm{O}), 3439.33(\mathrm{~N}-$ $\mathrm{H}$ ); Anal. Calcd. For $\mathrm{C}_{14} \mathrm{H}_{12} \mathrm{~N}_{2} \mathrm{O}_{5}$ : C 58.33, H 4.20, N 9.72 (\%). Found: C 58.31, H 4.23, N 9.75 (\%). 
Citation: Dhorajiya BD, Bhuva RG, Dholakiya BZ (2016) Design, Synthesis and Comparative Study of Anti-Microbial Activities on Barbituric Acid and Thiobarbituric Acid based Chalcone Derivatives Bearing the Pyrimidine Nucleus. Chem Sci J 7: 126. doi:10.4172/2150-3494.1000126

5-[3-(3-Methoxy-phenyl)-acryloyl]-2-thioxo-dihydropyrimidine-4,6-dione (5d'): Yellow Solid, M.W.304.32. Yield 62\%; m.p. $>250^{\circ} \mathrm{C} ;{ }^{1} \mathrm{H}$ NMR (DMSO- $\left.d_{6}\right): \delta 2.55(2 \mathrm{H}, \mathrm{s}, J=16.6$, trans$\mathrm{CH}=\mathrm{CH}), 3.69(1 \mathrm{H}, \mathrm{s},-\mathrm{CH}$ of pyrimidine ring at $\mathrm{C}-5), 3.76(3 \mathrm{H}, \mathrm{s}$, $\left.\mathrm{m}-\mathrm{OCH}_{3}\right), 6.78-7.08(2 \mathrm{H}, \mathrm{dd}, \mathrm{Ar}-\mathrm{H}), 7.90(1 \mathrm{H}, \mathrm{s}$, barbituric acid $\mathrm{NH})$, $7.93\left(1 \mathrm{H}, \mathrm{s}\right.$, barbituric acid NH); ${ }^{13} \mathrm{C}$ NMR (DMSO- $\left.d_{6}\right): \delta 58.08(\mathrm{C}-18)$, 78.85 (C-5), 113.30 (C-9), 113.47 (C-8), 122.95 (C-11, C-15), 129.27 (C-12, C-14), 131.17 (C-10, C-13), 157 (C-7), 162.66(C-4, C-6), 167.05 (C-2); FT-IR ( $\left.\mathrm{KBr}, \mathrm{cm}^{-1}\right): 1260.73(\mathrm{C}-\mathrm{SH}), 1603.57$ (C=C aromatic), $1687.97(\mathrm{C}=\mathrm{O}), 3343.39(\mathrm{~N}-\mathrm{H})$; Anal. Calcd. For $\mathrm{C}_{14} \mathrm{H}_{12} \mathrm{~N}_{2} \mathrm{O}_{4} \mathrm{~S}$ : C 55.25, H 3.97, N 9.21 (\%). Found: C 55.28, H 3.93, N 9.23 (\%).

5-[3-(3,4-Dimethoxy-phenyl)-acryloyl]pyrimidine-4,6-trione (5e): Yellow Solid, M.W. 318.28, Yield 83\%; m.p. 191-196 ${ }^{\circ} \mathrm{C} ;{ }^{1} \mathrm{H}$ NMR $\left(\mathrm{DMSO}-d_{6}\right): \delta 2.57(2 \mathrm{H}, \mathrm{s}, J=24$, trans- $\mathrm{CH}=\mathrm{CH}), 4.24(1 \mathrm{H}, \mathrm{s},-\mathrm{CH}$ of pyrimidine ring at $\mathrm{C}-5), 2.98\left(3 \mathrm{H}, \mathrm{s}, \mathrm{m}-\mathrm{OCH}_{3}\right), 3.09\left(3 \mathrm{H}, \mathrm{s}, \mathrm{p}-\mathrm{OCH}_{3}\right)$, 6.67-7.79 (2H, dd, Ar-H), $10.68(1 \mathrm{H}, \mathrm{s}$, barbituric acid NH), $10.89(1 \mathrm{H}$, s, barbituric acid NH); ${ }^{13} \mathrm{C}$ NMR (DMSO- $\left.d_{6}\right): \delta 57.36(\mathrm{C}-18, \mathrm{C}-17) 76.23$ (C-5), 113.96 (C-9), 115.17 (C-8), 123.55 (C-11, C-15), 128.78 (C-12, C-14), 130.87 (C-10, C-13), 157.32 (C-7), 161.69 (C-4, C-5), 168.56 (C2); FT-IR $\left(\mathrm{KBr}, \mathrm{cm}^{-1}\right): 1138.58(\mathrm{C}-\mathrm{O}), 1615.17(\mathrm{C}=\mathrm{C}$ aromatic), 1699.47 $(\mathrm{C}=\mathrm{O}), 3436.56(\mathrm{~N}-\mathrm{H})$; Anal. Calcd. $\mathrm{C}_{15} \mathrm{H}_{14} \mathrm{~N}_{2} \mathrm{O}_{6}: \mathrm{C} 56.60, \mathrm{H} 4.43, \mathrm{~N}$ 8.80 (\%). Found: C 56.58, H 4.26, N 8.82 (\%).

5-[3-(3,4-Dimethoxy-phenyl)-acryloyl]-2-thioxo-dihydropyrimidine-4,6-dione (5e'): Dark Yellow Solid, M.W. 334.35, Yield $74 \%$; m.p. $>250^{\circ} \mathrm{C}$; ${ }^{1} \mathrm{H}$ NMR (DMSO- $\left.d_{6}\right): \delta 2.53(2 \mathrm{H}, \mathrm{s}, J=16.3$, trans$\mathrm{CH}=\mathrm{CH}), 3.78(1 \mathrm{H}, \mathrm{s},-\mathrm{CH}$ of pyrimidine ring at $\mathrm{C}-5), 3.85(3 \mathrm{H}, \mathrm{s}$, $\left.\mathrm{m}-\mathrm{OCH}_{3}\right), 4.09\left(3 \mathrm{H}, \mathrm{s}, \mathrm{p}-\mathrm{OCH}_{3}\right), 6.75-7.18(2 \mathrm{H}, \mathrm{dd}, \mathrm{Ar}-\mathrm{H}), 7.76(1 \mathrm{H}$, s, barbituric acid $\mathrm{NH}), 7.95(1 \mathrm{H}, \mathrm{s}$, barbituric acid $\mathrm{NH}) ;{ }^{13} \mathrm{C} \mathrm{NMR}$ (DMSO- $d$ ): $\delta 58.08$ (C-18, C-17) 75.35 (C-5), 114.56 (C-9), 114.87 (C8), 122.95 (C-11, C-15), 129.27 (C-12, C-14), 131.17 (C-10, C-13), 158 (C-7), 162.66 (C-4, C-5), 167.05 (C-2); FT-IR $\left(\mathrm{KBr}, \mathrm{cm}^{-1}\right): 1261.69$ (C$\mathrm{SH}), 1607.12(\mathrm{C}=\mathrm{C}$ aromatic $), 1685.43(\mathrm{C}=\mathrm{O}), 3337.64(\mathrm{~N}-\mathrm{H})$; Anal. Calcd. $\mathrm{C}_{15} \mathrm{H}_{14} \mathrm{~N}_{2} \mathrm{O}_{5} \mathrm{~S}$ : C 53.88, H 4.22, N 8.38 (\%). Found: C 53.85, H $4.26, \mathrm{~N} 8.36(\%)$.

5-[3-(2-Chloro-phenyl)-acryloyl]pyrimidine-2,4,6-trione $\quad(5 \mathrm{f})$ : Yellow Solid, M.W. 292.67, Yield 69\%; m.p. 237-242 ${ }^{\circ}$ C; ${ }^{1} \mathrm{H}$ NMR $\left(\mathrm{DMSO}-d_{6}\right): \delta 2.59(2 \mathrm{H}, \mathrm{s}, J=28$, trans-CH=CH$), 4.25(1 \mathrm{H}, \mathrm{s},-\mathrm{CH}$ of pyrimidine ring at C-5), 6.59-7.94 $(2 \mathrm{H}, \mathrm{m}, \mathrm{Ar}-\mathrm{H}), 10.62(1 \mathrm{H}, \mathrm{s}$, barbituric acid $\mathrm{NH}), 10.81(1 \mathrm{H}$, s, barbituric acid $\mathrm{NH}) ;{ }^{13} \mathrm{C} \mathrm{NMR}$ $\left(\mathrm{DMSO}-d_{6}\right): \delta 75.31$ (C-5), 114.67 (C-9), 115.37 (C-8), 123.28 (C-13, C-14), 127.19 (C-12, C-15), 128.89 (C-11), 130.84 (C-10) 156.32 (C7), 161.68 (C-4, C-6), 167.28 (C-2); FT-IR $\left(\mathrm{KBr}, \mathrm{cm}^{-1}\right): 764.13(\mathrm{C}-\mathrm{Cl})$, $1137.69(\mathrm{C}-\mathrm{O}), 1620.23(\mathrm{C}=\mathrm{C}$ aromatic $), 1701.51(\mathrm{C}=\mathrm{O}), 3434.32(\mathrm{~N}-$ $\mathrm{H}$ ); Anal. Calcd. $\mathrm{C}_{13} \mathrm{H}_{9} \mathrm{ClN}_{2} \mathrm{O}_{4}$ : C 53.35, H 3.10, N 9.57 (\%). Found: $\mathrm{C}$ $53.38, \mathrm{H} 3.13$, N 9.55 (\%).

5-[3-(2-Chloro-phenyl)-acryloyl]-2-thioxo-dihydropyrimidine-4,6-dione (5f'): Brown Solid, M.W. 308.74, Yield 84\%; m.p. $>250^{\circ} \mathrm{C} ;{ }^{1} \mathrm{H}$ NMR (DMSO- $\left.d_{6}\right): \delta 2.51(2 \mathrm{H}, \mathrm{s}, J=16.2$, trans$\mathrm{CH}=\mathrm{CH}), 3.80(1 \mathrm{H}, \mathrm{s},-\mathrm{CH}$ of pyrimidine ring at $\mathrm{C}-5), 6.68-7.58(2 \mathrm{H}$, $\mathrm{m}, \mathrm{Ar}-\mathrm{H}), 7.74(1 \mathrm{H}, \mathrm{s}$, barbituric acid $\mathrm{NH}), 7.97(1 \mathrm{H}, \mathrm{s}$, barbituric acid $\mathrm{NH}) ;{ }^{13} \mathrm{C}$ NMR (DMSO- $\left.d_{6}\right): \delta 74.53$ (C-5), 113.26 (C-9), 113.59 (C-8), 122.92 (C-13, C-14), 126.51 (C-12, C-15), 129.39 (C-11), 131.78 (C10) 157.23 (C-7), 162.66 (C-4, C-6), 168.62 (C-2); FT-IR (KBr, $\left.\mathrm{cm}^{-1}\right)$ : 1254.71 (C-SH), 1615.42 (C=C aromatic), $1690.56(\mathrm{C}=\mathrm{O}), 3334.28(\mathrm{~N}-$ H); Anal. Calcd. $\mathrm{C}_{13} \mathrm{H}_{9} \mathrm{ClN}_{2} \mathrm{O}_{3} \mathrm{~S}: \mathrm{C} 50.57, \mathrm{H} 2.94$, N 9.07 (\%). Found: C 50.54, H 2.97, N 9.02 (\%).

5-[3-(4-Chloro-phenyl)-acryloyl]pyrimidine-2,4,6-trione (5g): Yellow Solid, M.W. 292.67, Yield 80\%; m.p. 258-263 ${ }^{\circ} \mathrm{C} ;{ }^{1} \mathrm{H}$ NMR $\left(\mathrm{DMSO}-d_{6}\right): \delta 2.56(2 \mathrm{H}, \mathrm{s}, J=28$, trans $-\mathrm{CH}=\mathrm{CH}), 4.23(1 \mathrm{H}, \mathrm{s},-\mathrm{CH}$ of pyrimidine ring at C-5), 6.71-7.24 $(2 \mathrm{H}, \mathrm{dd}, \mathrm{Ar}-\mathrm{H}), 10.65(1 \mathrm{H}, \mathrm{s}$, barbituric acid $\mathrm{NH}), 10.77(1 \mathrm{H}, \mathrm{s}$, barbituric acid $\mathrm{NH}) ;{ }^{13} \mathrm{C} \mathrm{NMR}$ $\left(\mathrm{DMSO}-d_{6}\right): \delta 77.72$ (C-5), 112.78 (C-9), 114.07 (C-8), 123.02 (C-13), 127.30 (C-14, C-15), 128.86 (C-11, C-12), 131.52 (C-10) 156.02 (C7), 163.07 (C-4, C-6), 167.57 (C-2); FT-IR (KBr, cm $\left.{ }^{-1}\right): 766.23$ (C-Cl), $1139.45(\mathrm{C}-\mathrm{O}), 1621.61(\mathrm{C}=\mathrm{C}$ aromatic), $1700.22(\mathrm{C}=\mathrm{O}), 3438(\mathrm{~N}-\mathrm{H})$; Anal. Calcd. $\mathrm{C}_{13} \mathrm{H}_{9} \mathrm{ClN}_{2} \mathrm{O}_{4}$ : C 53.35, H 3.13, N 9.57 (\%). Found (\%): C 53.33, H 3.10, N 9.58 (\%).

5- [3-(4-Chloro-phenyl)-acryloyl]-2-thioxo-dihydropyrimidine-4,6-dione (5g'): Dark brown Powder, M.W. 308.74, Yield $86 \%$; m.p. $>250^{\circ} \mathrm{C}$; ${ }^{1} \mathrm{H}$ NMR (DMSO- $d_{6}$ ): $\delta 2.53(2 \mathrm{H}, \mathrm{s}, J=16.4$, trans$\mathrm{CH}=\mathrm{CH}), 3.90(1 \mathrm{H}, \mathrm{s},-\mathrm{CH}$ of pyrimidine ring at $\mathrm{C}-5), 6.57-7.48(2 \mathrm{H}$, dd, Ar-H), $7.83(1 \mathrm{H}, \mathrm{s}$, barbituric acid $\mathrm{NH}), 7.97(1 \mathrm{H}, \mathrm{s}$, barbituric acid $\mathrm{NH}) ;{ }^{13} \mathrm{C}$ NMR (DMSO- $\left.d_{6}\right): \delta 76.37$ (C-5), 113.67 (C-9), 113.87 (C-8), 122.92 (C-13), 126.43 (C-14, C-15), 129.63 (C-11, C-12), 131.45 (C-10) 157.12 (C-7), 162.27 (C-4, C-6), 168.67 (C-2); FT-IR (KBr, cm $\left.{ }^{-1}\right): 1604$ $(\mathrm{C}=\mathrm{C}$ aromatic), $1684(\mathrm{C}=\mathrm{O}), 2527(\mathrm{C}=\mathrm{S}), 3331(\mathrm{~N}-\mathrm{H})$; Anal. Calcd. $\mathrm{C}_{13} \mathrm{H}_{9} \mathrm{ClN}_{2} \mathrm{O}_{3} \mathrm{~S}$ : C 50.57, H 2.94, N 9.07 (\%). Found (\%): C 50.51, H 2.99, N $9.04(\%)$.

5-[3-(3-Nitro-phenyl)-acryloyl]pyrimidine-2,4,6-trione (5h): Yellow Solid, M.W. 303.23, Yield 78\%; m.p. 203-208 ${ }^{\circ}$; ${ }^{1} \mathrm{H}$ NMR $\left(\right.$ DMSO- $\left.d_{6}\right): \delta 2.57(2 \mathrm{H}, \mathrm{s}, J=40$, trans- $\mathrm{CH}=\mathrm{CH}), 4.24(1 \mathrm{H}, \mathrm{s},-\mathrm{CH}$ of pyrimidine ring at C-5), 6.92-8.07 $(4 \mathrm{H}, \mathrm{m}, \mathrm{Ar}-\mathrm{H}), 10.68(1 \mathrm{H}, \mathrm{s}$, barbituric acid $\mathrm{NH}), 10.75(1 \mathrm{H}, \mathrm{s}$, barbituric acid $\mathrm{NH}) ;{ }^{13} \mathrm{C}$ NMR (DMSO-d): $\delta 77.32$ (C-7), 114.24 (C-9), 114.83 (C-8), 122.09 (C-13), 125.37 (C-12, C-14), 129.53 (C-11, C-15), 132.82 (C-10) 156.32 (C-7), 162.05 (C-4, C-6), 168.41 (C-2); FT-IR $\left(\mathrm{KBr}, \mathrm{cm}^{-1}\right): 1139.31\left(\mathrm{C}-\mathrm{NO}_{2}\right)$, $1351\left(\mathrm{C}-\mathrm{NO}_{2}\right), 1617(\mathrm{C}=\mathrm{C}$ aromatic), $1700(\mathrm{C}=\mathrm{O}), 3436(\mathrm{~N}-\mathrm{H})$; Anal. Calcd. $\mathrm{C}_{13} \mathrm{H}_{9} \mathrm{~N}_{3} \mathrm{O}_{6}$ : C 51.49, H 2.99, N 13.86 (\%). Found: C 51.46, H 2.97, N 13.89 (\%).

5-[3-(3-Nitro-phenyl)-acryloyl]-2-thioxo-dihydro-pyrimidine4,6-dione (5h'): Brown Solid, M.W. 319.29, Yield 88\%; m.p. $>250^{\circ} \mathrm{C}$; ${ }^{1} \mathrm{H}$ NMR (DMSO- $\left.d_{6}\right): \delta 2.52(2 \mathrm{H}, \mathrm{s}, J=16.7$, trans- $\mathrm{CH}=\mathrm{CH}), 3.65$ $(1 \mathrm{H}, \mathrm{s},-\mathrm{CH}$ of pyrimidine ring at $\mathrm{C}-5), 6.78-7.08(4 \mathrm{H}, \mathrm{m}, \mathrm{Ar}-\mathrm{H}), 7.86$ $(1 \mathrm{H}, \mathrm{s}$, barbituric acid $\mathrm{NH}), 7.97(1 \mathrm{H}, \mathrm{s}$, barbituric acid $\mathrm{NH}) ;{ }^{13} \mathrm{C} \mathrm{NMR}$ $\left(\mathrm{DMSO}-d_{6}\right): \delta 78.53(\mathrm{C}-7), 113.26(\mathrm{C}-9), 113.68$ (C-8), 121.29 (C-13), 126.43 (C-12, C-14), 129.58(C-11, C-15), 133.18 (C-10) 157.53 (C-7), 161.35 (C-4, C-6), 169.34 (C-2); FT-IR $\left(\mathrm{KBr}, \mathrm{cm}^{-1}\right): 1348\left(\mathrm{C}-\mathrm{NO}_{2}\right), 1609$ $(\mathrm{C}=\mathrm{C}$ aromatic), $1693(\mathrm{C}=\mathrm{O}), 2536(\mathrm{C}=\mathrm{S}), 3329(\mathrm{~N}-\mathrm{H})$; Anal. Calcd. $\mathrm{C}_{13} \mathrm{H}_{9} \mathrm{~N}_{3} \mathrm{O}_{5} \mathrm{~S}$ : C 48.90, H 2.84, N 13.16 (\%). Found: C 48.87, H 2.91, N $13.14(\%)$.

5-[3-(4-Nitro-phenyl)-acryloyl]pyrimidine-2,4,6-trione (5i): Orange Solid, M.W. 303.23, Yield 71\%; m.p. $209-213^{\circ} \mathrm{C} ;{ }^{1} \mathrm{H}$ NMR $\left(\right.$ DMSO- $\left.d_{6}\right): \delta 2.59(2 \mathrm{H}, \mathrm{s}, J=8$, trans- $\mathrm{CH}=\mathrm{CH}), 4.27(1 \mathrm{H}, \mathrm{s},-\mathrm{CH}$ of pyrimidine ring at C-5), 6.76-8.14 $(2 \mathrm{H}, \mathrm{dd}, \mathrm{Ar}-\mathrm{H}), 10.66(1 \mathrm{H}, \mathrm{s}$, barbituric acid $\mathrm{NH}), 10.75(1 \mathrm{H}, \mathrm{s}$, barbituric acid $\mathrm{NH}) ;{ }^{13} \mathrm{C}$ NMR (DMSO- $\left.d_{6}\right): \delta 76.03$ (C-7), $114.06(\mathrm{C}-9), 115.81(\mathrm{C}-8), 122.26(\mathrm{C}-13)$, 125.33 (C-12, C-14), 128.81 (C-11, C-15), 132.81 (C-10) 157.33 (C-7), 162.54 (C-4, C-6), 168.54 (C-2); FT-IR $\left(\mathrm{KBr}, \mathrm{cm}^{-1}\right): 1142.13(\mathrm{C}-\mathrm{O})$, $1355.12\left(\mathrm{C}-\mathrm{NO}_{2}\right), 1618(\mathrm{C}=\mathrm{C}$ aromatic), $1698.56(\mathrm{C}=\mathrm{O}), 3437(\mathrm{~N}-\mathrm{H})$; Anal. Calcd. $\mathrm{C}_{13} \mathrm{H}_{9} \mathrm{~N}_{3} \mathrm{O}_{6}$ : C 51.49, H 2.99, N 13.86 (\%). Found: C 51.48, H 2.96, N 13.87 (\%).

5-[3-(4-Nitro-phenyl)-acryloyl]-2-thioxo-dihydro-pyrimidine4,6-dione (5i'): Dark brown Powder, M.W. 319.29, Yield 82\%; m.p. $>250^{\circ} \mathrm{C} ;{ }^{1} \mathrm{H}$ NMR (DMSO- $\left.d_{6}\right): \delta 2.57(2 \mathrm{H}, \mathrm{s}, J=16.7$, trans- $\mathrm{CH}=\mathrm{CH})$, $3.70(1 \mathrm{H}, \mathrm{s},-\mathrm{CH}$ of pyrimidine ring at $\mathrm{C}-5), 6.68-7.28(2 \mathrm{H}, \mathrm{dd}, \mathrm{Ar}-\mathrm{H})$, $7.79(1 \mathrm{H}, \mathrm{s}$, barbituric acid $\mathrm{NH}), 8.03(1 \mathrm{H}, \mathrm{s}$, barbituric acid $\mathrm{NH}) ;{ }^{13} \mathrm{C}$ NMR (DMSO- $d_{6}$ ): $\delta 78.53$ (C-7), 113.26 (C-9), 113.68 (C-8), 121.29 (C- 
13), 126.43 (C-12, C-14), 129.58(C-11, C-15), 133.18 (C-10) 157.53 (C7), 161.35 (C-4, C-6), 169.34 (C-2); FT-IR $\left(\mathrm{KBr}, \mathrm{cm}^{-1}\right): 1353\left(\mathrm{C}-\mathrm{NO}_{2}\right)$, $1613(\mathrm{C}=\mathrm{C}$ aromatic), $1687(\mathrm{C}=\mathrm{O}), 2535(\mathrm{C}=\mathrm{S}), 3327(\mathrm{~N}-\mathrm{H})$; Anal. Calcd. $\mathrm{C}_{13} \mathrm{H}_{9} \mathrm{~N}_{3} \mathrm{O}_{6}$ : C 48.90, H 2.84, N 13.16 (\%). Found: C 48.92, H 2.81, N $13.17(\%)$.

5-(3-p-tolylacryloyl)pyrimidine-2,4,6(1H,3H,5H)-trione $\quad(5 \mathrm{j})$ : Yellow solid, M.W. 272.25, Yield $87 \%$; m.p. $182-187^{\circ} \mathrm{C} ;{ }^{1} \mathrm{H}$ NMR $\left(\mathrm{DMSO}-d_{6}\right): \delta 2.61(2 \mathrm{H}, \mathrm{s}, J=16$, trans- $\mathrm{CH}=\mathrm{CH}), 2.85\left(\mathrm{~s}, 3 \mathrm{H},-\mathrm{CH}_{3}\right)$, $4.22(1 \mathrm{H}, \mathrm{s},-\mathrm{CH}$ of pyrimidine ring at C-5), 6.75-8.14 $(2 \mathrm{H}, \mathrm{dd}, \mathrm{Ar}-\mathrm{H})$, $10.66(1 \mathrm{H}, \mathrm{s}$, barbituric acid $\mathrm{NH}), 10.72(1 \mathrm{H}, \mathrm{s}$, barbituric acid $\mathrm{NH})$; ${ }^{13} \mathrm{C}$ NMR (DMSO- $\left.d_{6}\right): \delta 77.37$ (C-5), $112.58(\mathrm{C}-9), 113.16(\mathrm{C}-8), 122.08$ (C-13), 127.40 (C-12, C-14), 128.17 (C-11, C-15), 133.02 (C-10), 157.56 (C-7), 167.52 (C-4, C-6), 168.64 (C-2); FT-IR (KBr, cm $\left.{ }^{-1}\right): 1137$ (C-O), 1614 (C=C aromatic), $1695(\mathrm{C}=\mathrm{O}), 3443(\mathrm{~N}-\mathrm{H})$; Anal. Calcd. $\left.\mathrm{C}_{14} \mathrm{H}_{12} \mathrm{~N}_{2} \mathrm{O}_{4}\right)$ : C $61.74, \mathrm{H} 4.44, \mathrm{~N} 10.29$ (\%). Found: C 61.74, H 4.46, N $10.31(\%)$.

dihydro-2-thioxo-5-(3-p-tolylacryloyl)pyrimidine-4,6(1H,5H)dione (5j'): Brown powder, M.W. 288.32, Yield 75\%; m.p. $>250^{\circ} \mathrm{C} ;{ }^{1} \mathrm{H}$ NMR (DMSO- $\left.d_{6}\right): \delta 2.54(2 \mathrm{H}, \mathrm{s}, J=16.3$, trans- $\mathrm{CH}=\mathrm{CH}), 3.69(1 \mathrm{H}, \mathrm{s},-\mathrm{CH}$ of pyrimidine ring at C-5), 6.48-7.38 $(2 \mathrm{H}, \mathrm{dd}, \mathrm{Ar}-\mathrm{H}), 7.08(1 \mathrm{H}, \mathrm{s}$, barbituric acid $\mathrm{NH}), 7.81(1 \mathrm{H}, \mathrm{s}$, barbituric acid $\mathrm{NH}) ;{ }^{13} \mathrm{C}$ NMR (DMSO- $\left.d_{6}\right): \delta 77(\mathrm{C}-$ 5), 113.45 (C-9), 113.76 (C-8), 121.78 (C-13), 126.34 (C-12, C-14), 129.87 (C-11, C-15), 132.22 (C-10), 158.25 (C-7), 166.15 (C-4, C-6), 169.26 (C2); FT-IR $\left(\mathrm{KBr}, \mathrm{cm}^{-1}\right): 1607(\mathrm{C}=\mathrm{C}$ aromatic), $1682(\mathrm{C}=\mathrm{O}), 2544(\mathrm{C}=\mathrm{S})$, 3345(N-H); Anal. Calcd. $\left.\mathrm{C}_{14} \mathrm{H}_{12} \mathrm{~N}_{2} \mathrm{O}_{3} \mathrm{~S}\right)$ : C 58.32, H 4.20, N 9.72 (\%). Found: C 58.29, H 4.24, N 9.76 (\%).

\section{5-(3-Naphthalen-2-yl-acryloyl)pyrimidine-2,4,6-trione}

(5k): Yellow Solid, M.W. 308.29, Yield 93\%; m.p. $187-193^{\circ} \mathrm{C}$; ${ }^{1} \mathrm{H}$ NMR $\left(\mathrm{DMSO}-d_{6}\right): \delta 2.59(2 \mathrm{H}, \mathrm{s}, J=32$, trans- $\mathrm{CH}=\mathrm{CH}), 4.24(1 \mathrm{H}, \mathrm{s},-\mathrm{CH}$ of pyrimidine ring at C-5), 6.68-7.71 $(7 \mathrm{H}, \mathrm{m}, \mathrm{Ar}-\mathrm{H}), 10.62(1 \mathrm{H}, \mathrm{s}$, barbituric acid $\mathrm{NH}), 10.78(1 \mathrm{H}, \mathrm{s}$, barbituric acid $\mathrm{NH}) ;{ }^{13} \mathrm{C} \mathrm{NMR}$ $\left(\mathrm{DMSO}-d_{6}\right)$ : $\delta 77.39$ (C-5), 113.58 (C-9), 113.37 (C-8), 121.05-134.52 (C-of Naphthalein ring), 156.13 (C-7), 164.73 (C-4, C-6), 169.54 (C2); FT-IR ( $\left.\mathrm{KBr}, \mathrm{cm}^{-1}\right)$ : 1141 (C-O), 1619.32 (C=C aromatic), 169812 $(\mathrm{C}=\mathrm{O}), 3439(\mathrm{~N}-\mathrm{H})$; Anal. Calcd. $\mathrm{C}_{17} \mathrm{H}_{12} \mathrm{~N}_{2} \mathrm{O}_{4}$ : C 66.23, H 3.92, N 9.09 (\%). Found: C 66.25, H 3.95, N 9.11 (\%).

5-(3-Naphthalen-2-yl-acryloyl)-2-thioxo-dihydro-pyrimidine4,6-dione (5k'): Dark brown Powder, M.W. 324.35, Yield 65\%; m.p. $>250^{\circ} \mathrm{C}$; ${ }^{1} \mathrm{H}$ NMR (DMSO- $\left.d_{6}\right): \delta 2.56(2 \mathrm{H}, \mathrm{s}, J=16.3$, trans- $\mathrm{CH}=\mathrm{CH})$, $3.83(1 \mathrm{H}, \mathrm{s},-\mathrm{CH}$ of pyrimidine ring at $\mathrm{C}-5), 6.75-7.14(7 \mathrm{H}, \mathrm{m}, \mathrm{Ar}-\mathrm{H})$, $7.89(1 \mathrm{H}, \mathrm{s}$, barbituric acid $\mathrm{NH}), 8.03(1 \mathrm{H}, \mathrm{s}$, barbituric acid $\mathrm{NH}) ;{ }^{13} \mathrm{C}$ NMR (DMSO- $\left.d_{6}\right): \delta 76$ (C-5), 114.52 (C-9), 114.77 (C-8), 122.95132.67 (C-of Naphthalein ring), 156.13 (C-7), 164.73 (C-4, C-6), 169.54 (C-2); FT-IR ( $\left.\mathrm{KBr}, \mathrm{cm}^{-1}\right): 1610(\mathrm{C}=\mathrm{C}$ aromatic), $1697(\mathrm{C}=\mathrm{O}), 2527$ $(\mathrm{C}=\mathrm{S}), 3338(\mathrm{~N}-\mathrm{H})$; Anal. Calcd. $\mathrm{C}_{17} \mathrm{H}_{12} \mathrm{~N}_{2} \mathrm{O}_{3} \mathrm{~S}: \mathrm{C} 62.95, \mathrm{H} 3.73, \mathrm{~N} 8.64$ (\%). Found: C 62.92, H 3.69, N 8.67 (\%).

\section{Biological assay}

In vitro antimicrobial activity evaluation: The synthesized barbituric acid and thiobarbituric acid derivatives 5 (a-a) and 5 (a'-k') were examined for antimicrobial activity against several bacteria (Bacillus subtilis MTCC 441, P. aeruginosa MTCC 1688, E. coli MTCC 443) and fungi (C. albicans MTCC 227) species using paper disc diffusion technique. The Muellere Hinton agar media were sterilized (autoclaved at $120^{\circ} \mathrm{C}$ for $30 \mathrm{~min}$ ), poured at uniform depth of $5 \mathrm{~mm}$ and allowed to solidify. The microbial suspension $\left(10^{5}\right.$ $\mathrm{CFU} / \mathrm{mL}$ ) (0.5 McFarland Nephelometery Standards) was streaked over the surface of media using a sterile cotton swab to ensure even growth of the organisms. The tested compounds were dissolved in dimethylsulfoxide to give solutions of $3.12-100 \mathrm{mg} / \mathrm{mL}$. Sterile filter paper discs measuring $6.25 \mathrm{~mm}$ in diameter (Whatman No. 1 filter paper), previously soaked in a known concentration of the respective test compound in dimethylsulfoxide were placed on the solidified nutrient agar medium that had been inoculated with the respective microorganism and the plates were incubated for $24 \mathrm{~h}$ at $(37 \pm 1)^{\circ} \mathrm{C}$. A control disc impregnated with an equivalent amount of dimethyl sulfoxide without any sample was also used and did not produce any inhibition. Ciprofloxacin (100 mg/ disc) were used as control drugs for antibacterial, Flucanazole and Griseofulvin for antifungal activity. MIC of the compound was determined by agar streak dilution method. A stock solution of the synthesized compound $(100 \mathrm{mg} / \mathrm{mL})$ in dimethylsulfoxide was prepared and graded quantities of the test compounds were incorporated in a specified quantity of molten sterile agar, i.e., nutrient agar for evaluation of antibacterial and sabouraud dextrose agar for antifungal activity, respectively. The medium containing the test compound was poured into a Petri dish at a depth of 4-5 mm and allowed to solidify under aseptic conditions. A suspension of the respective microorganism of approximately $10^{5} \mathrm{CFU} / \mathrm{mL}$ was prepared and applied to plates with serially diluted compounds with concentrations in the range of $3.12-100 \mathrm{mg} / \mathrm{mL}$ in dimethylsulfoxide and incubated at $(37 \pm 1)^{\circ} \mathrm{C}$ for $24 \mathrm{~h}$ (bacteria) or $48 \mathrm{~h}$ (fungi). The lowest concentration of the substance that prevents the development of visible growth is considered to be the MIC value.

Determination of zone of inhibition method: In vitro, antibacterial and antifungal activities were examined for all synthesized chalcone molecules. Antibacterial and antifungal activities of synthesized compounds against four pathogenic bacteria (one Gram positive and two Gram negative) and one pathogenic fungi were investigated by the Agar disk diffusion method [25-27]. Antimicrobial activity testing was carried out by using Agar cup method. Each synthesized compounds were dissolved in dimethyl sulfoxide (DMSO), sterilized by filtration using sintered glass filter and stored at $4^{\circ} \mathrm{C}$. For the determination of Zone of Inhibition, pure Gram positive, Gram negative and fungal strains were taken as a standard antibiotic for comparison of the results. All new synthesized molecules were screened for their antibacterial activities against the (Bacillus subtilis MTCC 441, E. coli MTCC 443, $P$. aeruginosa MTCC 1688) and one fungal strains (C. albicans MTCC 227). The sets of four dilutions $(25,50,100$ and $250 \mu \mathrm{g} / \mathrm{mL})$ and Standard drugs $25,50,100$ and $250 \mu \mathrm{g} / \mathrm{mL}$ ) were prepared in double distilled water using nutrient agar tubes. Muller Hinton sterile agar plates were seeded with indicator bacterial strains $\left(10^{8}\right.$ c.f.u.) and allowed to stay at $37^{\circ} \mathrm{C}$ for $3 \mathrm{~h}$. Control experiments were carried out under similar condition by using (Ciprofloxacin) and (Flucanazole and Greseofulvin) standard drugs for antibacterial and antifungal activity respectively. All of the plates were incubated at $37^{\circ} \mathrm{C}$ for 18 to $24 \mathrm{~h}$ for bacteria and at $28^{\circ} \mathrm{C}$ for 48 to $96 \mathrm{~h}$ for fungi. The zones of growth inhibition around the disks were measured after 18 to $24 \mathrm{~h}$ of in incubation at $37^{\circ} \mathrm{C}$ for bacteria and 48 to $96 \mathrm{~h}$ for fungi at $28^{\circ} \mathrm{C}$, respectively. The sensitivity of the microorganism species to the synthesized compounds were determined by measuring the sizes of inhibitory zones (including the diameter of disk) on the agar surface around the disks, and values $<10$ $\mathrm{mm}$ were considered as not active against microorganisms. The growth inhibition zone measured ranged from $10-23 \mathrm{~mm}$ for all the sensitive bacteria, and ranged from 10-25 mm for fungal strains (Table 1).

Structure activity relationship antibacterial study: A close investigation of the MIC values that all compounds exhibited a varied range of MIC (20-600 $\mu \mathrm{g} / \mathrm{ML})$ of antibacterial activity against three bacterial strains Bacillus subtilis MTCC 441, P. aeruginosa MTCC 1688 and E. coli MTCC 443. 
Effects of chalcones of barbituric acid and thiobarbituric acid on Bacillus subtilis MTCC-441: The MIC in $\mu \mathrm{g} / \mathrm{mL}$ and Zone of inhibition in $\mathrm{mm}$ for B. subtilis are given in Table 2. For compounds such $\mathbf{5 a}$ and 5a' without any substituent at ortho, meta and para position of the aryl ring attached as $\mathrm{HC}=\mathrm{HC}$ of chalcone motief showed MIC $600 \mu \mathrm{g} / \mathrm{mL}$ and $100 \mu \mathrm{g} / \mathrm{mL}$ respectively in this case the chalcones of thiobarbituric acid showed more MIC (Minimum Inhibition Concentration) than that of chalcones barbituric acid. In the case of compounds such $\mathbf{5 b}$ and $\mathbf{5 b}$ ' which have hydroxyl group at ortho position of the aryl ring at chalcone motif showed MIC $100 \mu \mathrm{g} / \mathrm{mL}$ and $62.5 \mu \mathrm{g} / \mathrm{mL}$ respectively in this case the chalcones of thiobarbituric acid showed more MIC than that of barbituric acid due to electron releasing nature of thioxo than the ketone. The compounds such $\mathbf{5} \mathbf{c}$ and $\mathbf{5} \mathbf{c}^{\prime}$ which have hydroxyl group at para position of the aryl ring at chalcone motif showed MIC $600 \mu \mathrm{g} / \mathrm{mL}$ and $100 \mu \mathrm{g} / \mathrm{mL}$ respectively in this case the chalcones of thiobarbituric acid showed more MIC than that of barbituric acid due electron releasing nature of thioxo than the ketone. A very interesting for the chalcones compounds such $\mathbf{5 d}$ and 5d' which have electron donating Methoxy group at para position of the aryl ring at chalcone motif showed MIC $20 \mu \mathrm{g} / \mathrm{mL}$ and $100 \mu \mathrm{g} / \mathrm{mL}$ respectively in this case the chalcones of barbituric acid showed more MIC than that of thiobarbituric acid owing to more electron releasing nature of ketonic than the thioxo group. On other hand compounds such $\mathbf{5 e}$ and $\mathbf{5} \mathbf{e}^{\mathbf{3}}$

\begin{tabular}{|c|c|c|c|c|c|}
\hline $\begin{array}{c}\text { Compound } \\
\text { Code }\end{array}$ & $\begin{array}{c}\text { For } \\
\text { compound }\end{array}$ & $\begin{array}{c}\text { For } \\
\text { compound }\end{array}$ & \multicolumn{3}{|c|}{$\begin{array}{c}\mathbf{R}_{\mathbf{1}}, \mathbf{R}_{2} \text { and } \mathbf{R}_{3} \text { are different } \\
\text { functional groups }\end{array}$} \\
\hline & $\mathbf{X = O}$ & $\mathbf{X = S}$ & $\mathbf{R}_{1}$ & $\mathbf{R}_{2}$ & $\mathbf{R}_{3}$ \\
\hline $5\left(\mathrm{a}-\mathrm{a}^{\prime}\right)$ & $5(\mathrm{a})$ & $5\left(\mathrm{a}^{\prime}\right)$ & $-\mathrm{H}$ & $-\mathrm{H}$ & $-\mathrm{H}$ \\
\hline $5\left(\mathrm{~b}-\mathrm{b}^{\prime}\right)$ & $5(\mathrm{~b})$ & $5\left(\mathrm{~b}^{\prime}\right)$ & $-\mathrm{H}$ & $-\mathrm{OH}$ & $-\mathrm{H}$ \\
\hline $5\left(\mathrm{c}-\mathrm{c}^{\prime}\right)$ & $5(\mathrm{c})$ & $5\left(\mathrm{c}^{\prime}\right)$ & $-\mathrm{H}$ & $-\mathrm{H}$ & $-\mathrm{OH}$ \\
\hline $5\left(\mathrm{~d}-\mathrm{d}^{\prime}\right)$ & $5(\mathrm{~d})$ & $5\left(\mathrm{~d}^{\prime}\right)$ & $-\mathrm{H}$ & $-\mathrm{H}$ & $-\mathrm{OCH}_{3}$ \\
\hline $5\left(\mathrm{e}-\mathrm{e}^{\prime}\right)$ & $5(\mathrm{e})$ & $5\left(\mathrm{e}^{\prime}\right)$ & $-\mathrm{H}$ & $-\mathrm{OCH}_{3}$ & $-\mathrm{OCH}_{3}$ \\
\hline $5\left(\mathrm{f}-\mathrm{f}^{\prime}\right)$ & $5(\mathrm{f})$ & $5\left(\mathrm{f}^{\prime}\right)$ & $-\mathrm{H}$ & $-\mathrm{Cl}$ & $-\mathrm{H}$ \\
\hline $5\left(\mathrm{~g}-\mathrm{g}^{\prime}\right)$ & $5(\mathrm{~g})$ & $5\left(\mathrm{~g}^{\prime}\right)$ & $-\mathrm{H}$ & $-\mathrm{H}$ & $-\mathrm{Cl}^{\prime}$ \\
\hline $5\left(\mathrm{~h}-\mathrm{h}^{\prime}\right)$ & $5(\mathrm{~h})$ & $5\left(\mathrm{~h}^{\prime}\right)$ & $-\mathrm{H}$ & $-\mathrm{NO}$ & $-\mathrm{H}$ \\
\hline $5\left(\mathrm{i}-\mathrm{i}^{\prime}\right)$ & $5(\mathrm{i})$ & $5\left(\mathrm{i}^{\prime}\right)$ & $-\mathrm{H}$ & $-\mathrm{H}$ & $-\mathrm{NO}_{2}$ \\
\hline $5\left(\mathrm{j}-\mathrm{j}^{\prime}\right)$ & $5(\mathrm{j})$ & $5\left(\mathrm{j}^{\prime}\right)$ & $-\mathrm{H}$ & $-\mathrm{H}$ & $-\mathrm{CH}_{3}$ \\
\hline $5\left(\mathrm{k}-\mathrm{k}^{\prime}\right)$ & $5(\mathrm{k})$ & $5\left(\mathrm{k}^{\prime}\right)$ & & \multicolumn{3}{|c|}{ Naphthalene Ring } \\
\hline
\end{tabular}

Table 1: List of compounds with their different functional groups.

\begin{tabular}{|c|c|c|c|c|c|c|c|c|c|c|}
\hline \multirow{4}{*}{$\begin{array}{c}\text { Compounds } \\
\text { code }\end{array}$} & \multicolumn{10}{|c|}{ Basillus subtilis MTCC-441 } \\
\hline & \multicolumn{5}{|c|}{ Barbituric acid } & \multicolumn{5}{|c|}{ Thiobarbitutric acid } \\
\hline & \multicolumn{4}{|c|}{ Zone of inhibition } & \multirow{2}{*}{$\begin{array}{c}\text { MIC } \\
\mu \mathrm{g} / \\
\mathrm{mL}\end{array}$} & \multicolumn{4}{|c|}{ Zone of inhibition } & \multirow{2}{*}{$\begin{array}{c}\text { MIC } \\
\mu \mathrm{g} / \\
\mathrm{mL}\end{array}$} \\
\hline & 25 & 50 & 100 & 250 & & 25 & 50 & 100 & 250 & \\
\hline 5 (a-a') & 10 & 10 & 10 & 10 & 600 & 10 & 12 & 14 & 18 & 100 \\
\hline 5 (b-b') & 10 & 10 & 11 & 12 & 100 & 11 & 13 & 17 & 19 & 62.5 \\
\hline $5\left(c-c^{\prime}\right)$ & 10 & 10 & 10 & 10 & 600 & 10 & 13 & 15 & 18 & 100 \\
\hline 5 (d-d') & 10 & 10 & 13 & 15 & 20 & 13 & 16 & 19 & 21 & 100 \\
\hline 5 (e-e') & 10 & 10 & 10 & 10 & 600 & 11 & 14 & 17 & 19 & 250 \\
\hline $5\left(f-f^{\prime}\right)$ & 10 & 10 & 10 & 10 & $>600$ & 10 & 13 & 16 & 19 & 62.5 \\
\hline $5\left(g-g^{\prime}\right)$ & 10 & 10 & 10 & 10 & $>600$ & 11 & 13 & 16 & 18 & 250 \\
\hline 5 (h-h') & 10 & 10 & 10 & 10 & $>600$ & 12 & 15 & 17 & 18 & 200 \\
\hline $5\left(i-i^{\prime}\right)$ & 10 & 10 & 12 & 12 & 60 & 11 & 13 & 17 & 19 & 100 \\
\hline $5\left(j-j^{\prime}\right)$ & 10 & 10 & 11 & 12 & 100 & 10 & 13 & 15 & 17 & 250 \\
\hline $5\left(k-k^{\prime}\right)$ & 10 & 10 & 10 & 10 & $>600$ & 10 & 12 & 15 & 18 & 200 \\
\hline $\begin{array}{l}\text { Standard } \\
\text { Drugs }\end{array}$ & \multicolumn{4}{|c|}{ Ciprofloxacin } & 05 & \multicolumn{4}{|c|}{ Ciprofloxacin } & 05 \\
\hline
\end{tabular}

Table 2: Comparative analysis data MIC and Zone of inhibition values of barbituric acid and thiobarbituric acid based chalcones with respect to Basillus subtilis MTCC-441. which have two electron donating Methoxy group at meta and para position of the aryl ring at chalcone motif showed MIC $600 \mu \mathrm{g} / \mathrm{mL}$ and $250 \mu \mathrm{g} / \mathrm{mL}$ respectively in this case the chalcones of thiobarbituric acid showed more MIC than that of barbituric acid due electron releasing nature of thioxo than the ketone and methoxy group at meta position reduces in the MIC. In the case of compounds such $\mathbf{5} \mathbf{f}$ and $\mathbf{5} \mathbf{f}^{\prime}$ which have electron with drawing chloro substituent at ortho position of the aryl ring at chalcone motif showed MIC $>600 \mu \mathrm{g} / \mathrm{mL}$ and $62.5 \mu \mathrm{g} / \mathrm{mL}$ respectively in this case the chalcones of thiobarbituric acid showed more MIC than that of barbituric acid due electron releasing nature of thioxo than the ketone. For compounds such $\mathbf{5 g}$ and $\mathbf{5 g}$ ' which have electron withdrawing chloro substituent at para position of the aryl ring at chalcone motif showed MIC $>600 \mu \mathrm{g} / \mathrm{mL}$ and $250 \mu \mathrm{g} / \mathrm{mL}$ respectively in this case the chalcones of thiobarbituric acid showed more MIC than that of barbituric acid due electron releasing nature of thioxo than the ketone and electron withdrawing nature of chloro group at para positin intensity in the MIC. In case of compounds such $\mathbf{5 h}$ and $\mathbf{5 h}$ ' which have strong electron withdrawing nitro substituent at meta position of the aryl ring at chalcone motif showed MIC $>600 \mu \mathrm{g} / \mathrm{mL}$ and $200 \mu \mathrm{g} / \mathrm{mL}$ respectively in this case the chalcones of thiobarbituric acid showed more MIC than that of barbituric acid due electron releasing nature of thioxo than the ketone and strong electron withdrawing nature of nitro group at meta position intensify in MIC in case of Bacillus subtilis MTCC441 as the bacterial strain. For the compounds such $\mathbf{5 i}$ and $\mathbf{5 i}$ ' which have strong electron withdrawing nitro substituent at para position of the aryl ring at chalcone motif showed MIC $60 \mu \mathrm{g} / \mathrm{mL}$ and $100 \mu \mathrm{g} / \mathrm{mL}$ respectively in this case the chalcones of barbituric acid showed more MIC than that of thiobarbituric acid due electron withdraing nature of Nitro group at para position and less electron withdrawing nature of ketone group than thioxo group. From compounds $\mathbf{5 h}-\mathbf{5 h}$ ' having one electron withdrawing group at meta position where as $\mathbf{5 i - 5 i}$ ' having two electron withdrawing group at meta and para position, the presence of two electron withdrawing increases in MIC values than one in at meta position. In the case of compounds such $\mathbf{5 j}$ and $\mathbf{5 j}$ ' which have strong electron donating methyl substituent at para position of the aryl ring at chalcone motif showed MIC $100 \mu \mathrm{g} / \mathrm{mL}$ and $250 \mu \mathrm{g} / \mathrm{mL}$ respectively in this case the chalcones of barbituric acid showed more MIC than that of thiobarbituric acid due electron releasing nature of methyl group at para position. In compounds such 5k-5k' naphthalene ring similar to $\mathbf{5 a - 5 a}$ ' where as phenyl ring attached as $\mathrm{HC}=\mathrm{HC}$ of chalcone motif showed MIC $>600 \mu \mathrm{g} / \mathrm{mL}$ and $200 \mu \mathrm{g} / \mathrm{mL}$ respectively in this case the chalcones of thiobarbituric acid showed more potency towards MIC than that of the barbituric acid. Thus from MIC values it has proved that the substituent at para position is responsible for Bacillus subtilis as the bacterial strains in case of barbituric acid based chalcones while rest of the compounds having substituent at ortho and meta position in this case chalcones of thiobarbituric acid were more potent than the chalcones of barbituric acid (Table 2; Figure 2).

Effects of Chalcones of barbituric acid and Thiobarbituric acid on P. aeruginosa MTCC 1688: From the MIC values of chalcones of thiobarbituric acid showed better potency towards $P$. aeriginosa as bacterial strain than that of the barbituric acid. In this case the thioketo group is intensifying the interaction with $P$. aeriginosa than the ketonic group. It proves that there is increase in interaction of $P$. aeriginosa and chalcone motief of thioxo group (Table 3; Figure 3).

Effects of chalcones of barbituric acid and thiobarbituric acid on E. coli MTCC 443: From MIC values it has proved that the substituents such as -Methoxy, -Nitro and -Methyl group at para position for $\mathbf{5 d}$ 


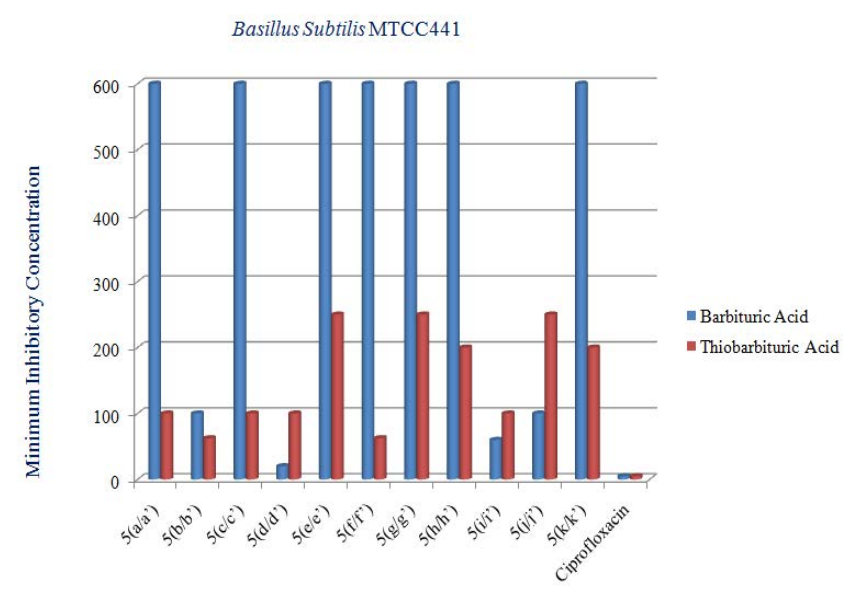

Compound Code

Figure 2: Comparative activity chart of barbituric acid and thiobarbituric acid based chalcones with respect to Bacillus with standard drugs.

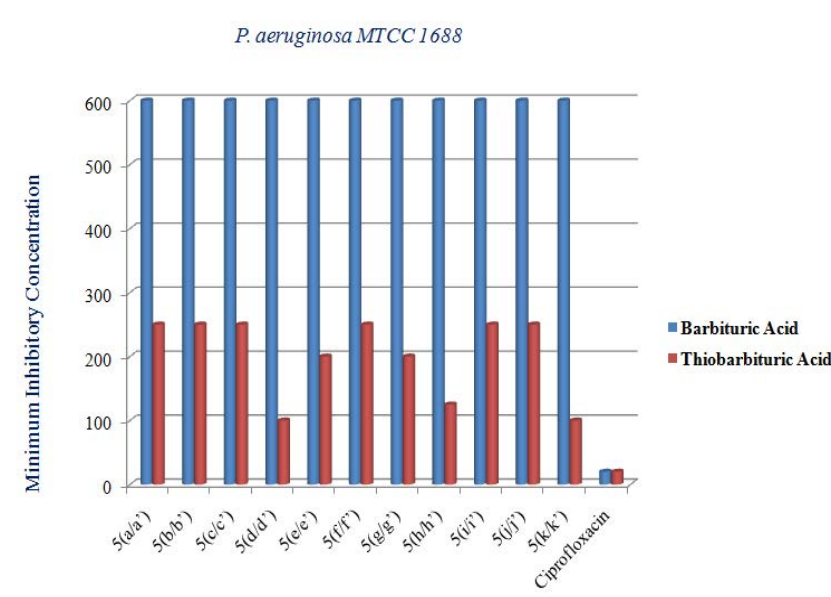

Compound Code

Figure 3: Comparative activity chart of barbituric acid and thiobarbituric acid based chalcones with respect to Pseudomonas aeruginosa with standard drugs.

$\mathbf{5 i}$ and $\mathbf{5} \boldsymbol{j}$ compounds respectively. chalcones of the barbituric acid are more potent than the chalcones of thiobarbituric acid viceversa (Table 4; Figure 4).

Effects of chalcones of barbituric acid and thiobarbituric acid on C. albicans MTCC 227 as the fungal strain: The MIC and Zone of inhibition for C. albicans are given in Table 5. From the MIC values it has been proved that the chalcone motief of thiobarbituric acid possessing various functional group at -Meta-Para, -Ortho and -Meta positions in $\mathbf{5} \mathbf{e}^{\mathbf{\prime}}, \mathbf{5} \mathbf{g}$ ' and $\mathbf{5} \mathbf{h}^{\prime}$ molecules shows better activity than the rest of molecules, where as the chalcones molecules of barbituric acid shows better activity independent of substituent and its positions (Figure 5 and Supplementary figures).

\section{Conclusion}

In Summary, from the antimicrobial study we conclude that, for gram positive bacterial strain $B$. subtilis Ortho and Para position of the chalcones of barbituric acid is more responsible where as in rest of the compounds thioxo group plays crucial role for the activity. For gram negative bacterial strains such as $P$. aeriginosa, thioxo group is more responsible for activity than the oxo group whereas in E. coli, the position of functional group at para positon is more responsible independent of nature of functional group. For fungal strain i.e., $C$. albican nature of functional group and position of functional group both factors are responsible for activity. The results unfold the way for investigation of new potential lead compounds for investigating antimicrobial activity. The present investigation showed that chalcone compounds of barbituric acid and thiobarbituric acid can be potential lead for development of new antibacterial and antifungal agents. On the basis of comparative analysis chalcones compound containing -thioxo group is more potent than the -oxo group. In future, pyrimidine based chalcones will use for the further development of new biological entity with reference to nature of functional group and its position.

\begin{tabular}{|c|c|c|c|c|c|c|c|c|c|c|}
\hline \multirow{4}{*}{$\begin{array}{l}\text { Compounds } \\
\text { code }\end{array}$} & \multicolumn{10}{|c|}{ P. aeruginosa MTCC-1688 } \\
\hline & \multicolumn{5}{|c|}{ Barbituric acid } & \multicolumn{5}{|c|}{ Thiobarbitutric acid } \\
\hline & \multicolumn{4}{|c|}{ Zone of inhibition } & \multirow{2}{*}{$\begin{array}{l}\text { MIC } \\
\mu \mathrm{g} / \\
\mathrm{mL}\end{array}$} & \multicolumn{4}{|c|}{ Zone of inhibition } & \multirow{2}{*}{$\begin{array}{l}\text { MIC } \\
\mu \mathrm{g} / \\
\mathrm{mL}\end{array}$} \\
\hline & 25 & 50 & 100 & 250 & & 25 & 50 & 100 & 250 & \\
\hline $5\left(a-a^{\prime}\right)$ & 10 & 10 & 10 & 10 & $>600$ & 10 & 13 & 15 & 17 & 250 \\
\hline 5 (b-b') & 10 & 10 & 10 & 10 & 600 & 11 & 13 & 17 & 19 & 250 \\
\hline $5\left(c-c^{\prime}\right)$ & 10 & 10 & 10 & 10 & 600 & 10 & 14 & 16 & 18 & 250 \\
\hline 5 (d-d') & 10 & 10 & 10 & 10 & $>600$ & 13 & 17 & 18 & 21 & 100 \\
\hline 5 (e-e') & 10 & 10 & 10 & 10 & $>600$ & 11 & 15 & 18 & 20 & 200 \\
\hline $5\left(f-f^{\prime}\right)$ & 10 & 10 & 10 & 10 & $>600$ & 10 & 14 & 17 & 18 & 250 \\
\hline $5\left(g-g^{\prime}\right)$ & 10 & 10 & 10 & 10 & $>600$ & 11 & 12 & 13 & 15 & 200 \\
\hline 5 (h-h') & 10 & 10 & 10 & 10 & 600 & 14 & 16 & 17 & 19 & 125 \\
\hline 5 (i-i') & 10 & 10 & 10 & 10 & 600 & 11 & 13 & 18 & 20 & 250 \\
\hline $5\left(j-j^{\prime}\right)$ & 10 & 10 & 10 & 10 & $>600$ & 10 & 12 & 16 & 17 & 250 \\
\hline $5\left(k-k^{\prime}\right)$ & 10 & 10 & 10 & 10 & $>600$ & 10 & 12 & 15 & 19 & 100 \\
\hline $\begin{array}{l}\text { Standard } \\
\text { Drugs }\end{array}$ & \multicolumn{4}{|c|}{ Ciprofloxacin } & 20 & \multicolumn{4}{|c|}{ Ciprofloxacin } & 20 \\
\hline
\end{tabular}

Table 3: Comparative activity analysis data MIC and Zone of inhibition values of barbituric acid and thiobarbituric acid based chalcones with respect to Pseudomonas aeruginosa.

\begin{tabular}{|c|c|c|c|c|c|c|c|c|c|c|}
\hline \multirow{4}{*}{$\begin{array}{l}\text { Compounds } \\
\text { code }\end{array}$} & \multicolumn{10}{|c|}{ E. coli MTCC -443} \\
\hline & \multicolumn{5}{|c|}{ Barbituric acid } & \multicolumn{5}{|c|}{ Thiobarbitutric acid } \\
\hline & \multicolumn{4}{|c|}{ Zone of inhibition } & \multirow{2}{*}{$\begin{array}{c}\text { MIC } \\
\mu \mathrm{g} / \\
\mathrm{mL}\end{array}$} & \multicolumn{4}{|c|}{ Zone of inhibition } & \multirow{2}{*}{$\begin{array}{c}\text { MIC } \\
\mu \mathrm{g} / \\
\mathrm{mL}\end{array}$} \\
\hline & 25 & 50 & 100 & 250 & & 25 & 50 & 100 & 250 & \\
\hline 5 (a-a') & 10 & 10 & 10 & 11 & 300 & 12 & 15 & 15 & 17 & 250 \\
\hline 5 (b-b') & 10 & 10 & 10 & 11 & 600 & 13 & 15 & 16 & 17 & 125 \\
\hline $5\left(\mathrm{c}-\mathrm{c}^{\prime}\right)$ & 10 & 10 & 10 & 11 & 600 & 15 & 17 & 16 & 17 & 250 \\
\hline $5\left(d-d^{\prime}\right)$ & 11 & 17 & 20 & 23 & 20 & 15 & 18 & 20 & 21 & 200 \\
\hline 5 (e-e') & 10 & 10 & 10 & 11 & 300 & 12 & 16 & 18 & 21 & 500 \\
\hline $5\left(f-f^{\prime}\right)$ & 10 & 10 & 10 & 11 & 300 & 13 & 14 & 15 & 16 & 250 \\
\hline $5\left(g-g^{\prime}\right)$ & 10 & 10 & 10 & 12 & 300 & 11 & 14 & 17 & 19 & 125 \\
\hline 5 (h-h') & 10 & 10 & 10 & 11 & 300 & 15 & 17 & 18 & 18 & 100 \\
\hline $5\left(i-i^{\prime}\right)$ & 12 & 13 & 18 & 21 & 20 & 12 & 15 & 19 & 23 & 250 \\
\hline $5\left(j-j^{\prime}\right)$ & 11 & 11 & 14 & 21 & 20 & 15 & 15 & 18 & 19 & 100 \\
\hline $5\left(k-k^{\prime}\right)$ & 10 & 10 & 10 & 11 & 300 & 14 & 16 & 18 & 21 & 250 \\
\hline $\begin{array}{l}\text { Standard } \\
\text { Drugs }\end{array}$ & \multicolumn{4}{|c|}{ Ciprofloxacin } & 10 & \multicolumn{4}{|c|}{ Ciprofloxacin } & 10 \\
\hline
\end{tabular}

Table 4: Comparative activity analysis data MIC and Zone of inhibition values of barbituric acid and thiobarbituric acid based chalcones with respect to Escherichia coli. 
Citation: Dhorajiya BD, Bhuva RG, Dholakiya BZ (2016) Design, Synthesis and Comparative Study of Anti-Microbial Activities on Barbituric Acid and Thiobarbituric Acid based Chalcone Derivatives Bearing the Pyrimidine Nucleus. Chem Sci J 7: 126. doi:10.4172/2150-3494.1000126

Page 8 of 9

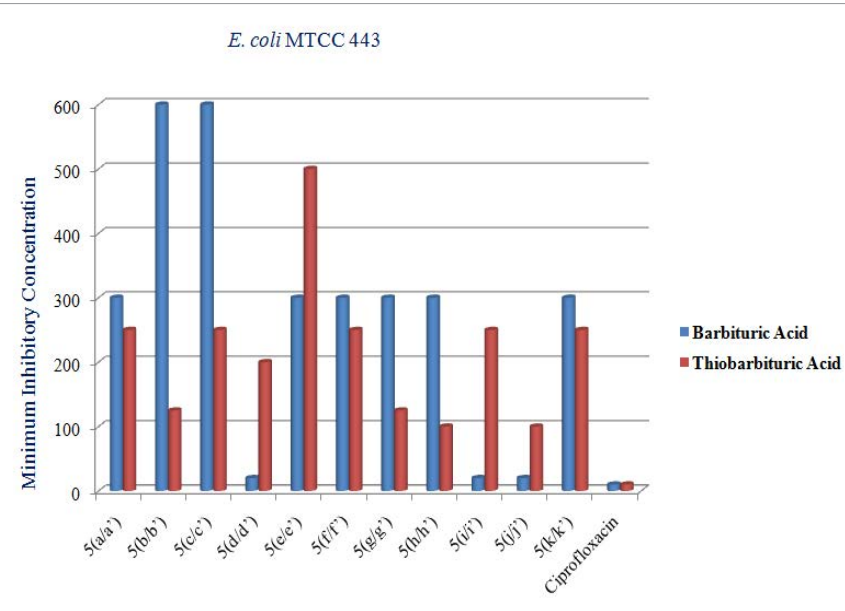

Compound Code

Figure 4: Comparative activitty chart of barbituric acid and thiobarbituric acid based chalcones with respect to Escherichia coli with standard drugs.

C. albicans MTCC 227

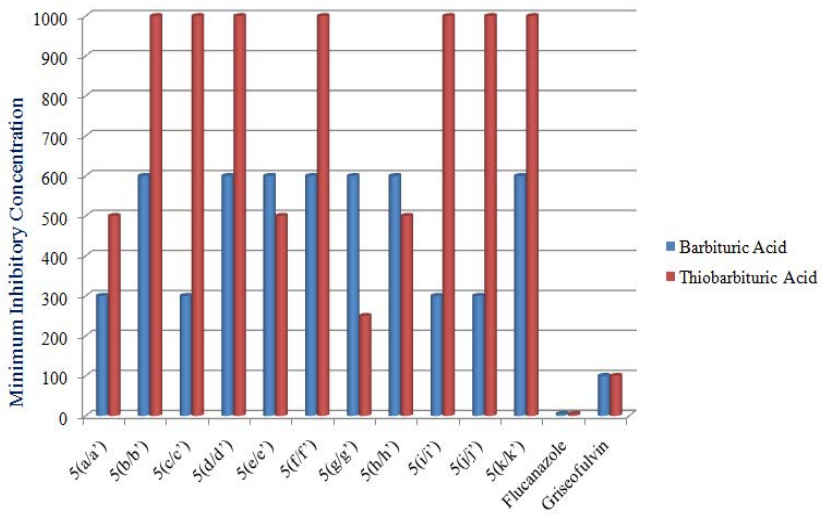

CompoundCode

Figure 5: Comparative activity chart of barbituric acid and thiobarbituric acid based chalcones with respect to Candida albicans with standard drugs.

From the comparative antimicrobial activity analysis of both of the series elucidated that it has been proved that the chalcone compounds containing -thioxo group are more potent towards bacterial as well as fungal strains than the -oxo group.

\section{Acknowledgements}

We are very much thankful to the Department of Applied Chemistry, SVNIT, Surat for providing laboratory facilities, financial Gujarat Council on Science and Technology (Project No. Gujcost/MRP/202042/12-13/04/1319). Mr. Paresh Kapopara and Mr. DP Rajani, Surat for antimicrobial activity determinations. For characterization, we are thankful to Centre of Excellence, Vapi, Mr. DM Thuma (Vice President) from Amoli Organic Pvt. Ltd., Baroda, Gujarat and Avtarsingh and Manishkumar from SAIF, Panjab University and Chandigarh, India.

\section{Author Contributions}

Bhaveshkumar D Dhoraijya conducted all the experimental section. Bhaveshkumar D Dhorajiya also wrote the manuscript which was revised by Bharatkumar Z Dholakiya. Both authors gave the approval to this final version of the manuscript.

\section{Conflicts of Interest}

The authors declare no conflict of interest.

\begin{tabular}{|c|c|c|c|c|c|c|c|c|c|c|}
\hline \multirow{4}{*}{$\underset{\text { code }}{\text { Compounds }}$} & \multicolumn{10}{|c|}{ C. albicans MTCC-227 } \\
\hline & \multicolumn{5}{|c|}{ Barbituric acid } & \multicolumn{5}{|c|}{ Thiobarbitutric acid } \\
\hline & \multicolumn{4}{|c|}{ Zone of inhibition } & \multirow{2}{*}{$\begin{array}{l}\text { MIC } \\
\mu \mathrm{g} / \\
\mathrm{mL}\end{array}$} & \multicolumn{4}{|c|}{ Zone of inhibition } & \multirow{2}{*}{$\begin{array}{c}\text { MIC } \\
\mu \mathrm{g} / \\
\mathrm{mL}\end{array}$} \\
\hline & 25 & 50 & 100 & 250 & & 25 & 50 & 100 & 250 & \\
\hline 5 (a-a') & 10 & 10 & 10 & 11 & 300 & 16 & 18 & 22 & 22 & 500 \\
\hline 5 (b-b') & 10 & 10 & 10 & 10 & 600 & 17 & 19 & 22 & 24 & 1000 \\
\hline $5\left(c-c^{\prime}\right)$ & 10 & 10 & 10 & 10 & 300 & 16 & 20 & 23 & 23 & $>1000$ \\
\hline $5\left(d-d^{\prime}\right)$ & 10 & 10 & 10 & 10 & 600 & 18 & 21 & 24 & 24 & $>1000$ \\
\hline 5 (e-e') & 10 & 10 & 10 & 10 & 600 & 20 & 24 & 25 & 25 & 500 \\
\hline $5\left(f-f^{\prime}\right)$ & 10 & 10 & 10 & 10 & 600 & 21 & 22 & 22 & 23 & 1000 \\
\hline 5 (g-g') & 10 & 10 & 10 & 10 & 600 & 20 & 21 & 22 & 23 & 250 \\
\hline 5 (h-h') & 10 & 10 & 10 & 10 & 600 & 21 & 22 & 23 & 24 & 500 \\
\hline $5\left(i-I^{\prime}\right)$ & 10 & 10 & 10 & 10 & 300 & 20 & 21 & 25 & 25 & 1000 \\
\hline $5\left(j-j^{\prime}\right)$ & 10 & 10 & 10 & 10 & 300 & 19 & 20 & 21 & 24 & 1000 \\
\hline $5\left(k-k^{\prime}\right)$ & 10 & 10 & 10 & 10 & $>600$ & 22 & 22 & 24 & 25 & $>1000$ \\
\hline \multirow{2}{*}{$\begin{array}{l}\text { Standard } \\
\text { Drugs }\end{array}$} & \multicolumn{4}{|c|}{ Flucanazole } & 05 & \multicolumn{4}{|c|}{ Flucanazole } & 05 \\
\hline & \multicolumn{4}{|c|}{ Griseofulvin } & 100 & \multicolumn{4}{|c|}{ Griseofulvin } & 100 \\
\hline
\end{tabular}

Table 5: Comparative analysis data MIC and Zone of inhibition values of barbituric acid and thiobarbituric acid based chalcones with respect to Candida albicans.

\section{References}

1. Siddiqui ZN, Musthafa TN, Ahmad A, Khan AU (2011) Thermal solventfree synthesis of novel pyrazolyl chalcones and pyrazolines as potential antimicrobial agents. Bioorg Med Chem Lett 21: 2860-2865.

2. Tenover FC, McDonald LC (2005) Vancomycin-resistant staphylococci and enterococci: epidemiology and control. Curr Opin Infect Dis 18: 300-305.

3. Cannito A, Perrissin M, Luu-Duc C, Huguet F, Gaultier C, et al. (1990) Synthesis and pharmacological activities of some 3-substituted thienopyrimidin-4-one-2thiones. Eur J Med Chem 25: 635-639.

4. Carmine AA, Brogden RN, Heel RC, Speight TM, Avery GS (1982) Trifluridine: a review of its antiviral activity and therapeutic use in the topical treatment of viral eye infections. Drugs 23: 329-353.

5. Dhorajiya BD, Patel JR, Malani MH, Dholakiya BZ (2012) Plant product (R) Roscovitine valuable inhibitor of CDKs as An anti-cancer agent. Der Pharmacia Sinica 3: 131-143.

6. Dhorajiya B, Malani M, Dholakiya M (2012) Extraction and preservation protocol of anti-cancer agents from marine world. Chem Scie J 38: 1-12.

7. Spaulding A, Rutherford GW, Siegfried N (2010) Stavudine or zidovudine in three-drug combination therapy for initial treatment of HIV infection in antiretroviral-naï ve individuals. Cochrane Database Syst Rev 4: CD008651.

8. Richards RME, Xing DKL (1992) Enhancement of antibacterial activity by p-aminobenzoic acid and sulphadiazine. Int J Pharm 82: 107-115.

9. Capan M, Mombo-Ngoma G, Makristathis A, Ramharter M (2010) Antibacterial activity of intermittent preventive treatment of malaria in pregnancy: comparative in vitro study of sulphadoxine- pyrimethamine, mefloquine, and azithromycin. Malar J 9: 303-307.

10. Kashman Y, Hirsh S, McConnell OJ, Ohtani I, Kusumi T, et al. (1989) A nove polycyclic guanidine alkaloid of marine origin. J Am Chem Soc 111: 8925-8926.

11. Basavaraja HS, Jayadevaiah KV, Mumtaz MH, Vijay Kumar MM, Basavara P (1989) Synthesis of novel piperazine and morpholine linked substituted pyrimidine derivatives as antimicrobial agents. J Pharm Sci Res 2: 5-12.

12. Holý A, Votruba I, Masojídková M, Andrei G, Snoeck R, et al. (2002) 6-[2-(Phosphonomethoxy) alkoxy] pyrimidines with antiviral activity. J Med Chem 45: 1918-1929.

13. Andres P, Marhold A (1996) A new synthesis of 5-trifluoromethyluracil Source J Fluorine Chem 77: 93-95

14. Reddick JJ, Saha S, Lee J, Melnick JS, Perkins J, et al. (2001) The mechanism of action of bacimethrin, a naturally occurring thiamin antimetabolite. Bioorg Med Chem Lett 11: 2245-2248.

15. Omar A, Eman Mostafa H, Neama A, Sherien I, Abd-Elmoez M (2010) Synthesis and Evaluation of Some Tetrahydropyrimidine Derivatives as Antimicrobia agents. Aus J Basic and Applied Sci 4: 27-36. 
Citation: Dhorajiya BD, Bhuva RG, Dholakiya BZ (2016) Design, Synthesis and Comparative Study of Anti-Microbial Activities on Barbituric Acid and Thiobarbituric Acid based Chalcone Derivatives Bearing the Pyrimidine Nucleus. Chem Sci J 7: 126. doi:10.4172/2150-3494.1000126

Page 9 of 9

16. Lawrence NJ, Patterson RP, Ooi LL, Cook D, Ducki S (2006) Effects of alphasubstitutions on structure and biological activity of anticancer chalcones. Bioorg Med Chem Lett 16: 5844-5848.

17. Gauthier C, Valdameri G, Terreux R, Kachadourian R, Day BJ, et al. (2012) Investigation of chalcones as inhibitors of $A B C G 2$ : identification of structural frameworks required for both potent inhibition and selectivity. J Med Chem 55: 3193-3200.

18. Jacobson RA (1937) Acyl ureas and process for the preparation thereof. US Patent, 2090594 A [C. A. 31, 7068].

19. Dhorajiya BD, Dholakiya BZ (2013) Green chemistry multi-component approach for $\mathrm{N}$-formylation and Knoevenagel condensation for synthesis of thiobarbiturates in aqueous system. Res Chem Intermed 41: 277-289.

20. Dhorajiya BD, Dholakiya BZ (2014) Green chemistry multicomponent protocol for formylation and Knoevenagel condensation for synthesis of (Z)-5arylaminomethylene pyrimidine 2, 4, 6-trione derivatives in water. Green Chem Lett Rev 7: 1-10.
21. Branko SJ, Donna MN (1937) Preparation of 5-formyl- and 5-acetylbarbituric acids, including the corresponding Schiff bases and phenylhydrazones. Tetrahedron Lett 42: 8435-8439.

22. Dhorajiya BD, Bhakhar BS, Dholakiya BZ (2013) Synthesis, Characterization, Solvatochromic properties and Antimicrobial evaluation of 5-acetyl-2-thioxodihydro-pyrimidine-4, 6-dione based Chalcones. Med Chem Res 22: 4075-4086.

23. Dhorajiya BD, Malani MH, Patel JR, Dholakiya BZ (2012) Antimicrobial activities of synthesized and characterized 5-acetyl pyrimidine-2, 4, 6- $(1 \mathrm{H}, 3 \mathrm{H}$, 5H)-trione based chalcone. Der Pharm Chem 4: 141-146.

24. Silverstein RM, Webster FX (1997) Spectrometric identification of organic compounds. 6th edn. New York, John Wiley \& Sons, pp: 79-223.

25. Alzoreky NS, Nakahara K (2003) Antibacterial activity of extracts from some edible plants commonly consumed in Asia. Int J Food Microbiol 80: 223-230.

26. Bauer AW, Kirby WM, Sherris JC, Turck M (1966) Antibiotic susceptibility testing by a standardized single disk method. Am J Clin Pathol 45: 493-496.

27. Rios JL, Recio MC, Villar A (1988) Screening methods for natural products with antimicrobial activity: a review of the literature. J Ethnopharmacol 23: 127-149. 